\title{
Intracluster light in the Virgo cluster: large scale distribution ${ }^{\star}$
}

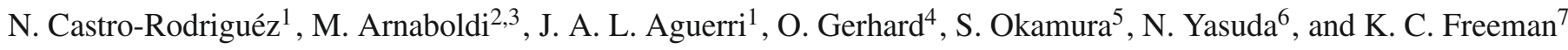 \\ 1 Instituto de Astrofísica de Canarias, C/O Vía Láctea s/n, 38200 La Laguna, Spain \\ e-mail: [ncastro; jalfonso]@iac.es \\ 2 European Southern Observatory, Karl-Schwarzschild-Strasse 2, 85748 Garching, Germany \\ e-mail: marnabol@eso.org \\ 3 INAF, Osservatorio Astronomico di Pino Torinese, 10025 Pino Torinese, Italy \\ 4 Max-Planck-Institut, Institut für Extraterrestrische Physik, Giessenbachstrasse, 85741 Garching, Germany \\ e-mail: gerhard@mpe.mpg.de \\ 5 Department of Astronomy and Research Center for the Early Universe, School of Science, University of Tokyo, \\ Tokyo 113-0033, Japan \\ e-mail: okamura@astron.s.u-tokyo.ac.jp \\ ${ }^{6}$ Institute for Cosmic Ray Research, University of Tokyo, Kashiwa, Chiba 277-8582, Japan \\ e-mail: yasuda@icrr.u-tokyo.ac.jp \\ 7 Research School of Astronomy and Astrophysics, Mount Stromlo Observatory, Cotter Road, Weston Creek, ACT 2611, Australia \\ e-mail: kcf@mso.anu.edu.au
}

Received 13 March 2008 / Accepted 11 August 2009

\section{ABSTRACT}

\begin{abstract}
Aims. The intracluster light (ICL) is a faint diffuse stellar component of clusters made of stars that are not bound to individual galaxies. We have carried out a large scale study of this component in the nearby Virgo cluster.

Methods. The diffuse light is traced using planetary nebulae (PNe). The surveyed areas were observed with a narrow-band filter centered on the [OIII] $\lambda 5007 \AA$ emission line redshifted to the Virgo cluster distance (the on-band image), and a broad-band filter (the off-band image). For some fields, additional narrow band imaging data corresponding to the $\mathrm{H} \alpha$ emission were also obtained. The PNe are detected in the on-band image due to their strong emission in the [OIII] $\lambda 5007 \AA$ Aine, but disappear in the off-band image. The contribution of Ly- $\alpha$ emitters at $z=3.14$ are corrected statistically using blank field surveys, when the $\mathrm{H} \alpha$ image at the field position is not available.

Results. We have surveyed a total area of 3.3 square degrees in the Virgo cluster with eleven fields located at different radial distances. Those fields located at smaller radii than 80 arcmin from the cluster center contain most of the detected diffuse light. In this central region of the cluster, the ICL has a surface brightness in the range $\mu_{\mathrm{B}}=28.8-30 \mathrm{mag} \mathrm{arsec}^{-2}$, it is not uniformly distributed, and represents about $7 \%$ of the total galaxy light in this area. At distances larger than 80 arcmin the ICL is confined to single fields and individual sub-structures, e.g. in the sub-clump B, the M 60/M 59 group. For several fields at 2 and 3 degrees from the Virgo cluster center we set only upper limits.

Conclusions. These results indicate that the ICL is not homogeneously distributed in the Virgo core, and it is concentrated in the high density regions of the Virgo cluster, e.g. the cluster core and other sub-structures. Outside these regions, the ICL is confined within areas of $\sim 100 \mathrm{kpc}$ in size, where tidal effects may be at work. These observational results link the formation of the ICL with the formation history of the most luminous cluster galaxies.
\end{abstract}

Key words. galaxies: clusters: individual: Virgo - galaxies: halos - planetary nebulae: general

\section{Introduction}

Galaxy clusters are the most massive gravitationally bound structures known in the Universe. In a volume of a few $\mathrm{Mpc}^{3}$, they contain a large number of galaxies that interact with each other, the hot gas and with the global cluster gravitational potential. Thus, in these high density regions the evolution of galaxies may be influenced by the environment.

With new numerical algorithms and fast computers, we are able to simulate large volumes of the Universe and follow the evolution of galaxies in different environments as a function of redshift. These simulations show that galaxies undergo

* Based on data collected with the $2.5 \mathrm{~m}$ Isaac Newton Telescope on La Palma, the Subaru telescope, operated by the National Astronomical Observatory of Japan, and the ESO/MPI $2.2 \mathrm{~m}$ telescope at La Silla, Chile, operated by ESO during observing runs $62 . \mathrm{N}-0248$ and $70 . \mathrm{B}$ 0086(A). transformations over time in cluster environments. They can evolve over short timescales (less than $1 \mathrm{Gyr}$ ) due to different mechanisms such as fast interactions between galaxies and with the cluster gravitational potential (Merritt 1984; Moore et al. 1996), gas stripping (Gunn \& Gott 1972; Quilis et al. 2000) and starvation (Larson et al. 1980; Bekki et al. 2002). Moreover, in the cluster central region, where the galaxy density is high, galaxies can also undergo important transformations over larger timescales due to galaxy mergers (Mihos 2004, and references therein). According to these results, galaxy evolution is not frozen at the earliest phases of the cluster formation, as described in earlier works (Richstone \& Malumuth 1983; Merritt 1984), but is still active today.

Hierarchical structure formation theories predict that massive galaxy clusters are built through the infall of matter, i.e. galaxies, groups and sub-clusters, along large scale filaments (White \& Rees 1978; West et al. 1995). Since mass accretion 
is still active today, nearby galaxy clusters may be at different epochs of their mass assembly, and they may still be in a unmixed state. The dynamical state of a galaxy cluster has been traditionally measured by determining the amount of substructure in the galaxy distribution and in the X-ray emitting hot intracluster medium, but the inferred results do not always agree (see Ramella et al. 2007, and references therein).

The gravitational forces acting on galaxies during cluster formation and its evolution unbind a fraction of their stars, which then end-up orbiting in the intracluster region. This unbound diffuse stellar component is the so-called intracluster light (ICL). Numerical simulations show that the amount of ICL in clusters depends on the cluster mass and dynamical state (Murante et al. 2004; Willman et al. 2004; Sommer-Larsen et al. 2005). A more massive, older, dynamically evolved cluster may contain a larger amount of ICL than a less massive or a dynamically younger system (Rudick et al. 2006; Murante et al. 2007). Furthermore, in a highly evolved dynamically old cluster the ICL morphology would be more diffuse, with relative few streams, while a dynamically young cluster dominated by groups still in the process of merging is likely to be dominated by ICL found in streams (Rudick et al. 2009). Therefore the study of the amount, distribution and kinematics of the ICL may provide information on the cluster accretion history and evolutionary state, as well as about the evolution of cluster galaxies.

The ICL has been mapped using several observational techniques, e.g. deep broadband imaging (Feldmeier et al. 2002, 2004b; Mihos et al. 2005; Zibetti et al. 2005; Krick \& Bernstein 2007), and the detection of individual stars associated with the diffuse stellar component, both red giant branch stars (Ferguson et al. 1998; Durrell et al. 2002; Williams et al. 2007) and intracluster planetary nebulae (ICPNe Arnaboldi et al. 1996, 2002, 2003; Feldmeier et al. 1998, 2003, 2004a; Aguerri et al. 2005, 2006). In particular, ICPNe are the only component of the ICL whose kinematics can be measured (Freeman et al. 2000; Arnaboldi et al. 2004; Gerhard et al. 2005, 2007; Doherty et al. 2009).

Several studies investigate the properties of the ICL in the core of the nearby Virgo cluster. Expanding on our earlier imaging work in the Virgo cluster core (Arnaboldi et al. 2002, 2003; Okamura et al. 2002; Aguerri et al. 2005), we have now completed a survey campaign of the ICL distribution on larger scales, outside the center of the Virgo cluster. In total, we have now covered more than 3 square degrees in Virgo, at eleven different positions in the cluster and at distances between 80 arcmin and some 100 arcmin from the Virgo cluster center. In several of the new fields, the ICL is at least two magnitudes fainter than in the core region. These new results are in agreement with observations of intermediate redshift clusters (Zibetti et al. 2005), and with the results of recent high resolution hydrodynamical simulations of cluster formation in a $\Lambda$ CDM universe (Murante et al. 2004; Rudick et al. 2006; Murante et al. 2007), which predict that the ICL is more centrally concentrated than cluster galaxies and that the largest portion of the ICL is formed during the assembly of the most luminous cluster galaxies.

In the following, we will present the results of a systematic and homogeneous ICPNe survey in regions outside the Virgo core and discuss the results jointly with ICPN survey data available for the Virgo cluster. In Sect. 2 we will present the data for the new fields in the Virgo cluster. The technique for the selection of ICPNe will be summarized in Sect. 3. The possible contamination of the ICPN catalog by background emission-line objects is quantified in Sect. 4. In Sect. 5 we use the number of detected ICPNe to infer the surface brightness of the light at the

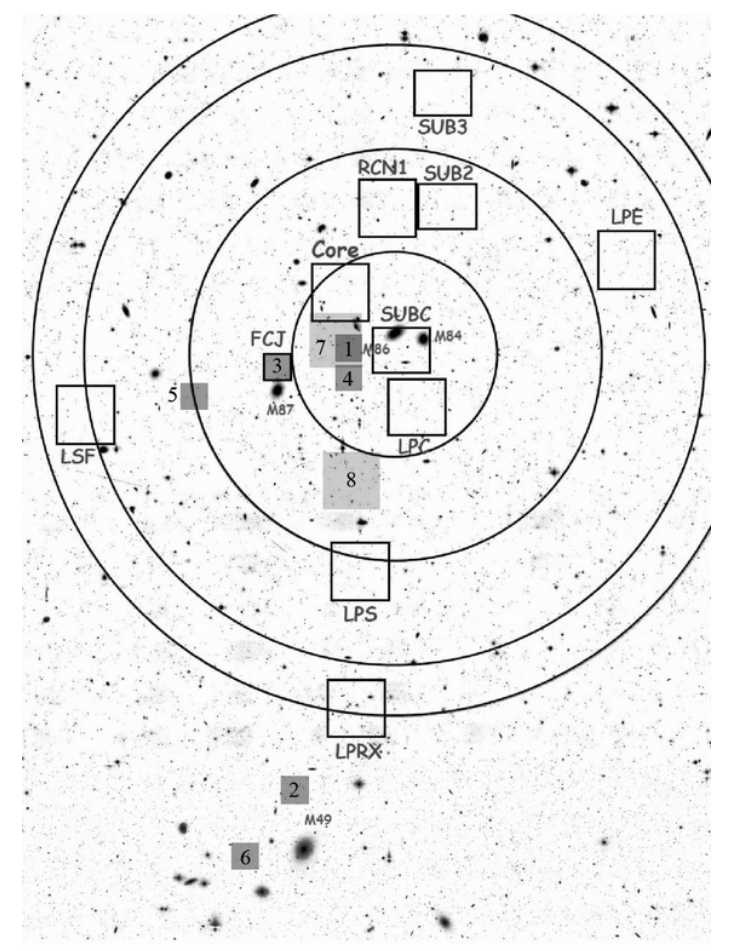

Fig. 1. Finding chart of the Virgo fields discussed in this paper. The circles indicate radial distances of $1,2,3$ and 3.5 degrees from the Virgo cluster center identified by Binggeli et al. (1987): $\alpha(\mathrm{J} 2000)=$ $12: 27: 49.95 \delta(\mathrm{J} 2000)=13: 01: 24.57$. Open squares indicate the field positions whose data were analyzed by our team. The area covered by each field is: $16 \times 16 \operatorname{arcmin}^{2}$ for the FCJ field, $34 \times 27 \mathrm{arcmin}^{2}$ for SUBC, SUB1 and SUB2 fields. The remaining fields have areas of $34 \times 34 \operatorname{arcmin}^{2}$ (Core, RCN1, LSF and LP* fields). The shaded fields indicate the areas surveyed by Feldmeier et al. (1998, 2003, 2004a); they are designated as F04- $n$ in the text, where $n$ is the number given in the respective shaded area on the figure.

fields' position and compare our results with previous works. We discuss the distribution of the ICL from the Virgo core to the outer distant fields at $\sim 200^{\prime}=0.8 \mathrm{Mpc}$, and its implications for current models for the formation of ICL in clusters, in Sect. 6. In Sect. 7 we set limits on the contribution from a possible homogeneous luminous density in the nearby Universe to our PN sample, and summarize our conclusions in Sect. 8.

In what follows we consider the center of the Virgo cluster at the position of Cluster A (Binggeli et al. 1987), at the peak of the luminosity density of all galaxies in the Virgo cluster, e.g. $\alpha(\mathrm{J} 2000)=12: 27: 49.95 \delta(\mathrm{J} 2000)=13: 01: 24.57$. We adopt a distance of $15 \mathrm{Mpc}$ to the Virgo cluster center, thus $1^{\prime \prime}=72.7 \mathrm{pc}$.

\section{Observations and data reduction}

Previous ICPN surveys in the Virgo cluster were carried out mostly in the Virgo core (Feldmeier et al. 1998, 2003, 2004a; Arnaboldi et al. 2002, 2003; Aguerri et al. 2005) and around M 49 (Feldmeier et al. 2004a).

We have now collected additional data in several fields located at larger radial distances from the center of the Virgo cluster. In total we have now six new fields, which in addition to those surveyed in Aguerri et al. (2005) cover a total area of 3.3 square degrees. The Virgo cluster SDSS image and the location of the eleven fields (five fields surveyed in previous studies by our group and the six new ones) are shown in Fig. 1. 
Table 1. Summary of the field positions, filter characteristics and exposure times for the narrow-band (on-band) images.

\begin{tabular}{|c|c|c|c|c|c|c|c|c|c|c|}
\hline \multirow[b]{2}{*}{ Field } & \multirow[b]{2}{*}{$\begin{array}{l}\text { RA(J2000) } \\
\text { (hh mm ss) }\end{array}$} & \multirow[b]{2}{*}{$\begin{array}{c}\operatorname{Dec}(\mathrm{J} 2000) \\
\left({ }^{\circ},{ }^{\prime}\right)\end{array}$} & \multirow[b]{2}{*}{$\begin{array}{l}\lambda_{\mathrm{c}} \\
(\AA)\end{array}$} & \multicolumn{2}{|c|}{ ["OIII] } & \multirow[b]{2}{*}{$\begin{array}{l}S_{F W H M} \\
(\operatorname{arcsec})\end{array}$} & \multicolumn{3}{|c|}{$\overline{\mathrm{H} \alpha}$} & \multirow[b]{2}{*}{$\begin{array}{l}S_{F W H M} \\
(\operatorname{arcsec})\end{array}$} \\
\hline & & & & $\begin{array}{c}F W H M \\
(\AA)\end{array}$ & $\begin{array}{c}\text { Exposure } \\
\text { (s) }\end{array}$ & & $\begin{array}{l}\lambda_{\mathrm{c}} \\
(\AA)\end{array}$ & $\begin{array}{c}F W H M \\
(\AA)\end{array}$ & $\begin{array}{c}\text { Exposure } \\
\text { (s) }\end{array}$ & \\
\hline Core & 122748 & +131846 & 5023 & 80 & 24000 & 1.20 & - & - & - & - \\
\hline FCJ & 123039 & +123810 & 5027 & 44 & 18000 & 1.40 & - & - & - & - \\
\hline LPC & 122532 & +121439 & 5027 & 60 & 27000 & 1.40 & - & - & - & - \\
\hline SUBC & 122547 & +124358 & 5021 & 74 & 3600 & 0.68 & 6607 & 101 & 8728 & 0.70 \\
\hline LPRX & 122800 & +093000 & 5027 & 60 & 27000 & 1.80 & - & - & - & - \\
\hline LPE & 121727 & +134130 & 5027 & 60 & 27000 & 1.30 & - & - & - & - \\
\hline LPS & 122734 & +104342 & 5027 & 60 & 27000 & 1.30 & - & - & - & - \\
\hline LSF & 123833 & +120841 & 5023 & 80 & 33000 & 1.08 & - & - & - & - \\
\hline RCN1 & 122613 & +140803 & 5023 & 80 & 24000 & 1.20 & - & - & - & - \\
\hline SUB2 & 122413 & +140901 & 5021 & 74 & 3600 & 1.00 & 6607 & 101 & 9000 & 1.10 \\
\hline SUB3 & 122432 & +152121 & 5021 & 74 & 3600 & 1.00 & 6607 & 101 & 9000 & 1.05 \\
\hline
\end{tabular}

The fields listed above the horizontal line are located within 80 arcmin of the Virgo cluster center; the data reduction is described in previous work (FCJ: Arnaboldi et al. 2002; SUBC: Arnaboldi et al. 2003; Core and LPC: Aguerri et al. 2005). The fields listed below the horizontal line are at distances between 80 and 215 arcmin of Virgo cluster center.

Table 2. Summary of the filter characteristics and exposure times for the broad-band (off-band) images in the various fields.

\begin{tabular}{|c|c|c|c|c|c|c|c|c|c|c|c|c|}
\hline \multirow[b]{2}{*}{ Field } & \multicolumn{4}{|c|}{$\bar{B} B$-band } & \multicolumn{4}{|c|}{$\bar{V} V$-band } & \multicolumn{4}{|c|}{$\bar{R} R$-band } \\
\hline & $\begin{array}{c}\lambda_{\mathrm{c}} \\
(\AA)\end{array}$ & $\begin{array}{c}F W H M \\
(\AA)\end{array}$ & $\begin{array}{l}\text { Exposure } \\
\text { (s) }\end{array}$ & $\begin{array}{c}S_{F W H M} \\
(\operatorname{arcsec})\end{array}$ & $\begin{array}{c}\lambda_{\mathrm{c}} \\
(\AA)\end{array}$ & $\begin{array}{c}F W H M \\
(\AA)\end{array}$ & $\begin{array}{l}\text { Exposure } \\
\text { (s) }\end{array}$ & $\begin{array}{c}S_{F W H M} \\
(\operatorname{arcsec})\end{array}$ & $\begin{array}{c}\lambda_{\mathrm{c}} \\
(\AA)\end{array}$ & $\begin{array}{c}F W H M \\
(\AA)\end{array}$ & $\begin{array}{l}\text { Exposure } \\
\text { (s) }\end{array}$ & $\begin{array}{c}S_{F W H M} \\
(\operatorname{arcsec})\end{array}$ \\
\hline Core & - & - & - & - & 5395 & 894 & 2400 & 1.20 & - & - & - & - \\
\hline FCJ & - & - & - & - & 5300 & 267 & 6000 & 1.4 & - & - & - & - \\
\hline LPC & 4407 & 1022 & 5400 & 1.50 & - & - & - & - & - & - & - & - \\
\hline SUBC & - & - & & & 5500 & 956 & 900 & 1.00 & 6500 & 1130 & 720 & 0.86 \\
\hline LPRX & 4407 & 1022 & 15600 & 1.60 & - & - & - & - & - & - & - & - \\
\hline LPE & 4407 & 1022 & 15600 & 1.50 & - & - & - & - & - & - & - & - \\
\hline LPS & 4407 & 1022 & 15600 & 1.50 & - & - & - & - & - & - & - & - \\
\hline LSF & - & - & - & - & 5395 & 894 & 14300 & 1.10 & - & - & - & - \\
\hline RCN1 & - & - & - & - & 5395 & 894 & 2400 & 1.20 & - & - & - & - \\
\hline SUB2 & - & - & - & - & 5500 & 956 & 3600 & 1.10 & 6500 & 1130 & 1440 & 0.80 \\
\hline SUB3 & - & - & - & - & 5500 & 956 & 3600 & 0.80 & 6500 & 1130 & 1440 & 0.90 \\
\hline
\end{tabular}

We indicate also the areas surveyed by Feldmeier et al. (1998, 2003, 2004a) and the name of the brightest Virgo galaxies. The results of the survey for ICPNe in the LPRX, LPE, LPS, LSF, SUB2, SUB3 field are presented here for the first time. In Tables 1 and 2 we give the eleven fields' IDs and locations.

Three of the new fields were observed during two observing runs in March 2002 and 2004 with the Wide Field Camera (WFC) on the $2.5 \mathrm{~m}$ Isaac Newton Telescope (INT) at Roque de los Muchachos Observatory (La Palma, see Tables 1 and 2). We refer to them hereafter as the LPRX, LPE and LPS fields. The images from this camera consists of a mosaic of four CCDs with a total field of view of $34^{\prime} \times 34^{\prime}$. The pixel scale is 0.333 , and the mean readout noise and gain of the detectors are 6.1 ADU pixel ${ }^{-1}$ and $2.8 \mathrm{e}^{-} \mathrm{ADU}^{-1}$, respectively. We imaged these fields through a $60 \AA$ wide filter centred at $5027 \AA$, which contains the wavelength of the [OIII] $\lambda 5007 \AA$ emission at the redshift of the Virgo cluster. We will refer to this narrowband filter as the "on-band" filter (see Table 1). We also took an additional broad-band image per field through a filter centred on the $B$ band ( $4407 \AA$ ) and $1022 \AA$ wide. The broad-band filter is referred to as the "off-band" (see Table 2). The total exposures were $27000 \mathrm{~s}$ for the on-band images and $15600 \mathrm{~s}$ for the offband.

In April 2004 we acquired another field (LSF) with the Wide Field Imager (WFI) on the ESO/MPI $2.2 \mathrm{~m}$ telescope located at La Silla Observatory (Chile). The full image from this camera consists of a mosaic of eight CCD images, covering $34^{\prime} \times 34^{\prime}$ on the sky. Each CCD has a pixel scale of 0.238 , and an average readout noise and gain of 4.5 $\mathrm{ADU}_{\text {pixel }}{ }^{-1}$ and $2.2 \mathrm{e}^{-} \mathrm{ADU}^{-1}$, respectively. The field was imaged through an on-band $80 \AA$ wide filter, centred at $5023 \AA$. In addition we also imaged this field through an off-band $V$-band $894 \AA$ wide filter, centred at $5395 \AA$ (see Tables 1 and 2).

From January to April 2004 two fields in the Virgo Cluster (SUB2 and SUB3) were imaged with the Suprime-Cam $10 \mathrm{k} \times 8 \mathrm{k}$ mosaic camera, at the prime focus of the $8.2 \mathrm{~m}$ Subaru telescope (Miyazaki et al. 2002). The field of view of these images covers an area of $34^{\prime} \times 27^{\prime}$, with a pixel size of 0.2 . The fields were imaged through two on-band and two off-band filters, the standard $V$ and $R$ filters (see Tables 1 ands 2).

The new fields at larger cluster distances from M 87 are complementary to those studied in the Virgo cluster core; their parameters are reported in Tables 1 and 2. The data reduction and calibration of the new fields, LPRX, LPE, LPS and LSF, follow the procedure described in Arnaboldi et al. (2002, hereafter Paper I). The data reduction is performed using the MSCRED package in IRAF $^{1}$. Calibrations are done using several Landolt fields for the off-band filters, and spectrophotometric stars for the on-bands. Fluxes are normalized to the AB magnitude system, following Theuns \& Warren (1997). A detailed description of the calibration steps and the relation between the AB magnitudes and the $m(5007)$ [OIII] magnitude introduced by Jacoby (1989) is given in Paper I. Table 3 shows the conversion constant $(K)$ between the two magnitude systems: $m(5007)=m_{\mathrm{AB}}+K$.

The data for the additional fields observed with the SuprimeCam at the SUBARU telescope (SUB2 and SUB3)

\footnotetext{
1 For a detailed discussion of the mosaic data reduction, we refer the reader to Alcala et al. (2002).
} 
Table 3. Limiting magnitudes of the fields in the on-band, off-band filter, and the $K$ constant.

\begin{tabular}{cccc}
\hline \hline Field & $m_{\text {lim }}(5007)$ & $m_{\text {blim }}$ & $K$ \\
\hline Core & 27.21 & 24.75 & 2.51 \\
FCJ & 27.01 & 24.58 & 2.50 \\
LPC & 27.52 & 25.40 & 3.02 \\
SUBC & 28.10 & - & 2.49 \\
\hline LPRX & 27.49 & 25.05 & 3.02 \\
LPE & 27.83 & 25.93 & 3.02 \\
LPS & 27.69 & 25.91 & 3.02 \\
LSF & 28.20 & 26.32 & 2.51 \\
RCN1 & 26.71 & 25.63 & 2.51 \\
SUB2 & 27.99 & 26.81 & 2.49 \\
SUB3 & 27.74 & 26.65 & 2.49 \\
\hline
\end{tabular}

are processed using the standard data reduction package developed for SuprimeCam data. The photometric calibrations for the SUB2 and SUB3 data are obtained using Landolt fields for the broad bands, and spectrophotometric stars for the narrow bands ${ }^{2}$.

For completeness, Tables 1 and 2 provide a summary of the field positions, filter characteristics and exposure times for onband and off band exposures for the surveyed areas in the Virgo core. Throughout this work we will use the notation $m_{\mathrm{n}}$ and $m_{\mathrm{b}}$ to refer the on-band and off-band magnitudes of the objects in the $\mathrm{AB}$ photometric system.

\section{Detection and selection of ICPN candidates}

In this work, the diffuse light in a Virgo cluster field is measured by the number density of detected PNe. The PNe are identified via their strong emission in the [OIII] $\lambda 5007 \AA$ line and, in the two SUB2, SUB3 field, may be confirmed by the additional detection of the associated $\mathrm{H} \alpha$ emission. The observational techniques used for PNe detection have been successfully applied in the Virgo cluster, in nearby groups (Leo; Castro-Rodríguez et al. 2003), and in compact groups (HCG44; Aguerri et al. 2006). In a few Virgo fields we carried out spectroscopic follow-up, resulting in a large fraction (up to $80 \%$ for some fields) of spectroscopically confirmed PNe (Arnaboldi et al. 2004; Doherty et al. 2009).

\section{1. [OIII] "on-off" band survey of ICPNe - Catalog selection with the color magnitude diagram (CMD)}

The ICPN candidates in the fields LPRX, LPE, LPS and LSF were identified using the on-off band technique, pioneered by Ciardullo et al. (1989) and automated for large areas by Arnaboldi et al. (2002, Paper I), using selection criteria based on color excess discussed by Theuns \& Warren (1997). On the basis of the strong line emission of a PN at the [OIII] $\lambda 5007 \AA$ line, the photometric ICPN candidates can be identified as point-like sources detected in the [OIII] image, the on-band and, because of their faint continuum, they cannot be detected in the off-band image. The on-band filter is a few tens of $\AA$ wide ( 60 or $80 \AA$, see Tables 1 and 2) centred at the wavelength of the redshifted [OIII]5007 A emission of the PN population at a given distance. In Paper I, this technique was standardized and automatized to select ICPNe in wide field images. We give a brief summary here.

\footnotetext{
${ }^{2}$ Zero points for the Subaru data are $z_{0, \mathrm{H} \alpha}=24.0, z_{0,[\mathrm{OIII}]}=24.6$, and $z_{0, V+R}=27.8$ for a $1 \mathrm{~s}$ exposure.
}

We detect all objects in the on-band images. We then perform aperture photometry with SExtractor (Bertin \& Arnouts 1996) on the on-band image at the sources' position, and in the corresponding aperture in the off-band image. All point-like sources detected in the on-band image are placed in a color-magnitude diagram (CMD), $m_{\mathrm{n}}-m_{\mathrm{b}}$ vs. $m_{\mathrm{n}}$, and are classified according to positions in this diagram. The ICPN candidates are pointlike objects with no detected continuum emission and observed $E W$ greater than the color excess curve corresponding to $E W=$ $100 \AA$ and convolved with the photometric error, see Paper I for more details. Figure 2 shows the CMDs for the LPRX, LPE, LPS, and LSF fields; the selected ICPN candidates are indicated by asterisk and square symbols.

The magnitude limit of our emission-line catalog is evaluated using Monte Carlo simulations. We distribute a large sample of point-like objects $(\sim 10000)$ randomly on the scientific images. We then perform the photometry of the objects using the same procedure as for the real emission sources. The limiting magnitude is then defined as the magnitude at which $50 \%$ of the simulated population is retrieved. In Table 3 we list the limiting magnitudes for the on-band image in the $m(5007)$ photometric system, and for the off-band magnitude in the Johnson system. We list also the conversion constant $K$ between the ABsystem and $m(5007)$. According to Table 3 the "shallowest" field is RCN1 $\left(m_{\lim }(5007)=26.71\right)$ and the deepest field is LSF $\left(m_{\lim }(5007)=28.20\right)$, with $m_{\lim }(5007)$ fainter than 27.0 for all the other survey fields.

At the distance of $15 \mathrm{Mpc}$, the bright cut-off of the PN luminosity function (PNLF) falls at the apparent magnitude of $m(5007)=26.38^{3}$ which is brighter than the limiting magnitude in all surveyed fields.

On the basis of the CMD selection, we detect 9, 20, 7 and 23 emission-line point-like objects brighter than the $m_{\lim }(5007)$ in LPRX, LPE, LPS, and LSF, respectively. These emission sources include ICPN candidates and background galaxies. In Appendix A we give the coordinates and $m$ (5007) magnitudes of these sources.

\section{2. [OIII] and $\mathrm{H} \alpha$ survey of ICPNe - Catalog selection with the 2-color diagram (2-CD)}

A more secure selection of the ICPN candidates is possible in the in the SUB2 and SUB3 fields, using the additional information from the $\mathrm{H} \alpha$ images which eliminates the contamination from background galaxies (Okamura et al. 2002; Mihos et al. 2009). The selection procedure is described in Arnaboldi et al. (2003, hereafter Paper II), on the basis of the instrumental magnitudes measured for sources in the fields, normalized to $1 \mathrm{sec}$ exposure.

We use SExtractor for the detection of point-like objects in SUB2 and SUB3 [OIII] on band images. Once the objects are extracted, we measure fluxes at the sources' positions in the $\mathrm{H} \alpha, V$ and $R$ band images in matching apertures. We then use a two-color diagram (2-CD), [OIII]-H $\alpha$ vs. [OIII]- $(V+R)$, for the selection of the ICPN candidates. In this 2-CD, continuum sources, single-line and two-line emitters, either PNe or compact HII regions (Gerhard et al. 2002), occupy different regions of the plane. We establish some ad-hoc selection rules for this specific set of images, and the detection/photometry described above. Simulated populations of PNe, single line emitters, and continuum sources are constructed in the $2-\mathrm{CD}$, and are used

\footnotetext{
3 This is computed using the absolute magnitude for the PNLF bright cut-off of $M_{5007}^{*}=-4.5$ (Ciardullo et al. 2002a) and a distance modulus of 30.88 .
} 

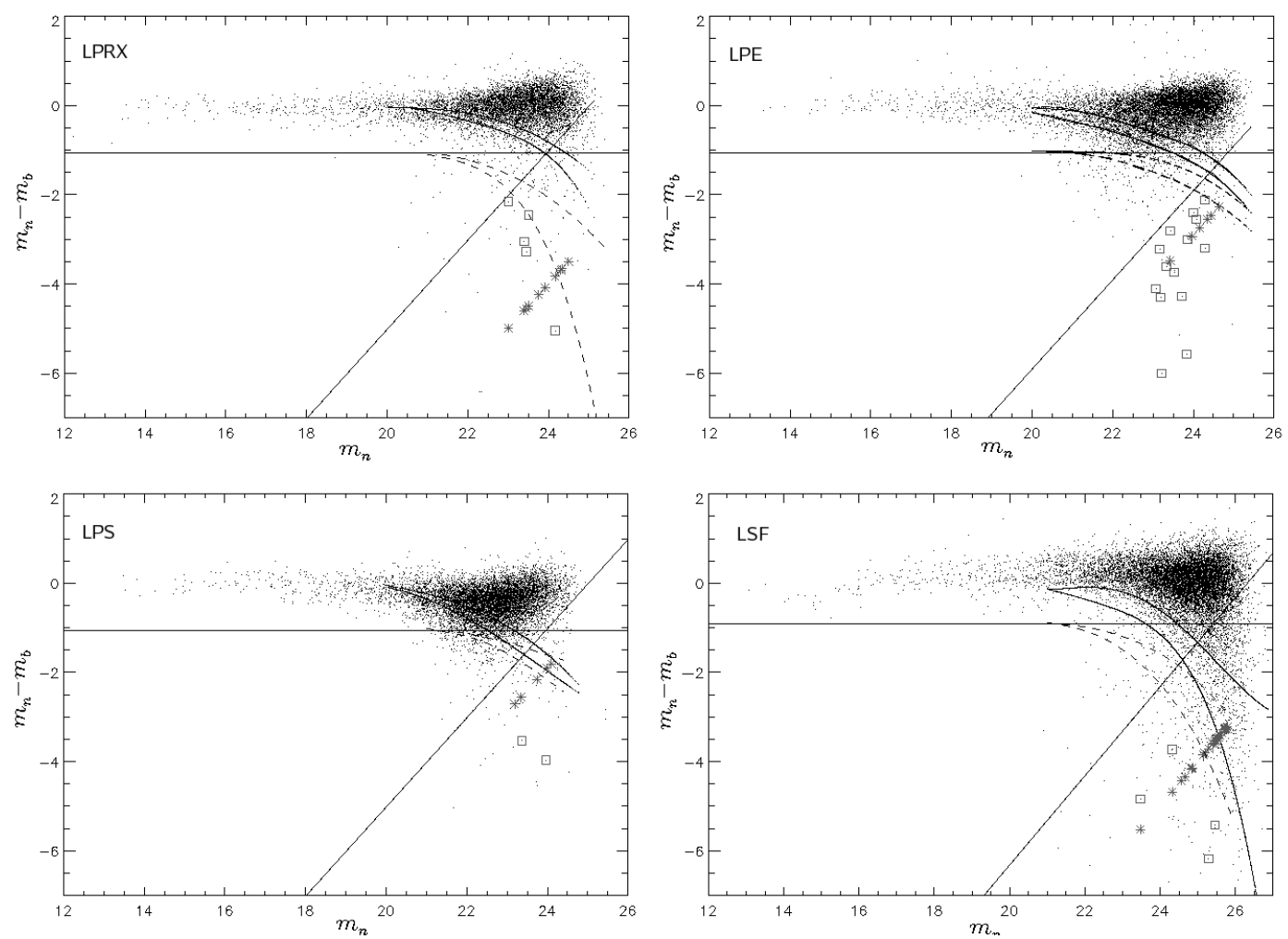

Fig. 2. Color-magnitude diagrams (CMDs) for all sources in the LPRX, LPE, LPS and LSF fields. The horizontal lines indicate the color excess of emission line objects with an observed $E W=100 \AA$. The inclined continuous lines show the $m_{\mathrm{b}}$ magnitude corresponding to $1.0 \sigma$ above the sky, in the off-band images. The curved lines limit the regions where $99 \%$ and $99.9 \%$ of the simulated continuum objects would fall in this diagram, given the photometric errors. The dashed lines limit those regions where $84 \%$ and $97.5 \%$ of the simulated objects with color excess relative to an observed $E W=100 \AA$ would fall in this diagram, as a function of magnitude and the photometric errors. The asterisks represent point-like sources with off-band magnitudes fainter than the detection limit in the off-band image as measured by SExtractor; the squares represent point-like objects with an off-band emission fainter than the limiting magnitude in the off-band image.

to outline the regions inhabited by the different kind of objects. Differently from Paper II, we have the zero points of the photometric calibration for the narrow bands, [OIII] and $\mathrm{H} \alpha$, and for the broad band images, $V$ and $R$, and the selection criteria for the different classes of objects translate into these color constraints:

- the simulated PN population consists of point-like objects with a color excess in [OIII], e.g. $m_{\mathrm{n}}-m_{(V+R)}<-1.3$, and have line ratios $[\mathrm{OIII}] /(\mathrm{H} \alpha+[\mathrm{NII}])$ larger than 3 (Ciardullo et al. 2002a), because they populate the brighter part of the PNLF. This line ratio translates into an [OIII] vs. H $\alpha$ color excess of $m_{\mathrm{n}}-m_{\mathrm{H} \alpha}<-1$. Therefore, the ICPN-like candidates are point-like sources detected in both [OIII] and $\mathrm{H} \alpha$ images, whose colors are $m_{\mathrm{n}}-m_{(V+R)}<-1.3$ and $m_{\mathrm{n}}-m_{\mathrm{H} \alpha}<-1$; the simulated objects are plotted as open squares in Fig. 3;

- the continuum sources are simulated with a flat spectral energy distribution which translates into $m_{\mathrm{n}}-m_{\mathrm{H} \alpha}=m_{\mathrm{n}}-$ $m_{(V+R)}=0$ colors; these are plotted as open triangles in Fig. 3;

- single line emitters in [OIII] are those objects detected in the [OIII] image, have upper limits in either $\mathrm{H} \alpha$ or in the $V+R$ continuum image, or have no detections in those images. These are plotted as asterisks in Fig. 3. Bright $m_{\mathrm{n}}$ sources without $\mathrm{H} \alpha$ emission are background, large $E W$ Ly- $\alpha$ emitters, while fainter $m_{\mathrm{n}}$ sources without $\mathrm{H} \alpha$ emission can be either PNe or background objects. Because of the different detection limits and noise in the $\mathrm{H} \alpha$ and $V+R$ images, they are scattered in the different regions of the 2-CD. See Paper II for additional details;

- compact HII regions are sources with faint continuum, $m_{\mathrm{n}}-$ $m_{\mathrm{H} \alpha}>0$ and $m_{\mathrm{n}}-m_{(V+R)}<0$, because of the stronger $\mathrm{H} \alpha$ line emission.

The different classes of simulated objects are added to the scientific images and then recovered using the same automatic procedure as for the real objects. Figure 3 shows the 2-CDs of the simulated objects for the SUB2 and SUB3 fields.

The limiting magnitude of the [OIII], $\mathrm{H} \alpha, V$ and $R$ band images are listed in Table 3. The $m_{\mathrm{lim}}(5007)$ for the on-band [OIII] images of the SUB2 and SUB3 fields is 1.5 mag fainter than the apparent magnitude of the PNLF bright cut-off at $15 \mathrm{Mpc}$ distance. The 2-CDs and CMDs of the emission objects in SUB2 and SUB3 images are shown in Fig. 4.

\subsection{Comparison between the two selection procedures}

For the SUB2/SUB3 fields, we can compare the two methods for detecting ICPNe either with CMD and 2-CDs. Figure 4 shows the CMD for these two fields: we have obtained 13 and $11 \mathrm{ob}-$ jects in SUB2 and SUB3 brighter than the $m_{\mathrm{lim}}(5007)$ in these fields, that are classified as ICPNe on the basis of color excess criteria in the CMD. Figure 4 also shows the distribution of those objects in the 2-CDs. It turns out that most of the objects classified as ICPNe in the CMDs are located in the region occupied by single line emitter in the 2-CDs, i.e. they do have a detectable 

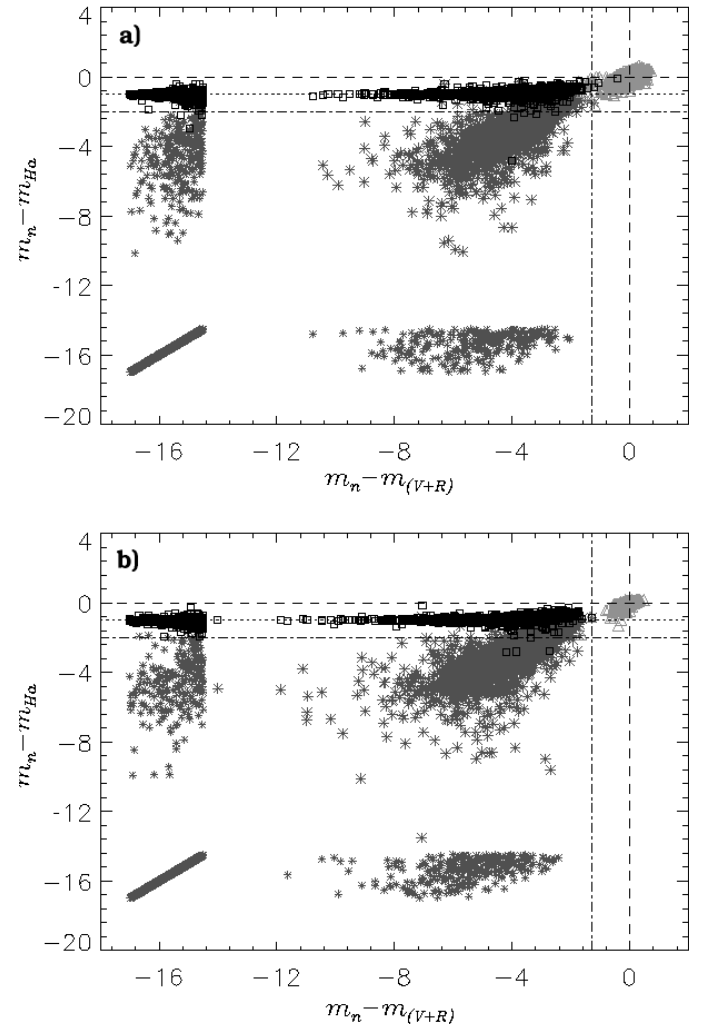

Fig. 3. Color-color diagrams $\left(m_{\mathrm{n}}-m_{\mathrm{H} \alpha}\right)$ vs. $\left(m_{\mathrm{n}}-m_{(V+R)}\right)$ of simulated objects for SUB2 a) and SUB3 b). The different symbols represent: continuum objects (open triangles), single line emitters (asterisks), and PNe (open squares). Vertical and horizontal dashed lines show objects with $m_{\mathrm{n}}-m_{\mathrm{H} \alpha}=m_{\mathrm{n}}-m_{(V+R)}=0$. The vertical dashed-dotted line represents objects with $m_{\mathrm{n}}-m_{(V+R)}=1.3$. The horizontal dotted and dash-dotted lines show objects with $m_{\mathrm{n}}-m_{\mathrm{H} \alpha}=-1$ and -2 for SUB2 and SUB3. The continuum and/or $\mathrm{H} \alpha$ magnitudes of the simulated objects with no continuum and/or no $\mathrm{H} \alpha$ detection were set arbitrarily to 40 .

$\mathrm{H} \alpha$ emission at the position of the [OIII] source on the $\mathrm{H} \alpha$ image. Four objects, one in SUB2 and three in SUB3, which are ICPN candidates according to the CMDs, are also two-line emitters according to the 2-CD.

From the 2-CDs, we conclude that we detect one ICPN in SUB2 and three ICPNe in SUB3 brighter than the limiting magnitudes in [OIII] and $\mathrm{H} \alpha$; the four ICPNe are indicated by open diamonds in the right panel of Fig. 4. Furthermore, no HII compact regions are detected in these two fields.

In empty fields, the sources selected on the basis of a color excess in a narrow band, e.g. in the 4500-5000 A wavelength interval, vs. a broad band filter selected catalog, are most probably Ly- $\alpha$ background galaxies at redshift $\simeq 3$. 1 . The identification of these sources as Ly- $\alpha$ emitters has been confirmed with spectroscopic follow-up (Kudritzki et al. 2000; Castro-Rodríguez et al. 2003; Gawiser et al. 2007; Rauch et al. 2008). Therefore when only a single deep [OIII] image is available to produce the catalog of point-like emission line objects for a given Virgo field, we need to statistically subtract the background population of the Ly- $\alpha$ emitters to select the over-density of objects associated with the PN population.

We now discuss the statistical subtraction of such a background population in the Virgo cluster fields in Sect. 4.

\section{On the nature of the emission-line sources in the Virgo outer fields}

In addition to a population of ICPNe at the Virgo cluster distance, in the CMD-selected source list with color excess, we expect a contribution from misclassified faint continuum objects, [OII] emitters at intermediate redshift and Ly- $\alpha$ background galaxies at $z \approx 3.1$. We discuss the contribution from faint continuum sources in Sect. 4.1, and background galaxies in Sect. 4.2 with the goal of selecting the most likely PN population at these field positions.

\subsection{Contamination by faint continuum objects}

It is possible that our catalogs contain misclassified faint continuum objects. Because of the photometric errors and a steeply rising luminosity function, a non-negligible number of faint continuum objects, which would have $m_{\mathrm{n}}$ fainter than the limiting magnitude in the on-band image, may end up having a measured $m_{\mathrm{n}}$ brighter than $m_{\mathrm{lim}}(5007)$. As the same time, due to the photometric errors, the measured off-band magnitude may be fainter than expected, resulting in a color excess for these continuum sources as for the ICPNe. Given the large number of stars in the field, the number of such sources can be significant, if the off-band is not sufficiently deep.

We call this the "spillover" effect in Paper III (see for additional details). The number of continuum objects that "spilled over" can be estimated from simulations of point-like continuum objects with a LF extrapolated to 3 mag fainter than $m_{\text {lim }}(5007)$ and distributed randomly on the scientific frames. We then detect the simulated objects with the same criteria as for the real ones, and determine the number of simulated objects brighter than $m_{\mathrm{lim}}$ (5007) in regions of the CMD where the ICPN photometric candidates are located. After scaling the simulations to the number of observed continuum objects, the final numbers of spilled-over contaminants brighter than $m_{\text {lim }}(5007)$ of the image are found to be zero in all fields. This result comes about because of the very deep off-band images acquired for these fields (see the discussion in Paper III).

\subsection{Contribution from background galaxies}

Background emission-line galaxies are detected in [OIII] on-band photometric surveys (Ciardullo et al. 2002b; Castro-Rodríguez et al. 2003; Gronwall et al. 2007). According to the central wavelength and limiting flux of the on-band images, the surveys are sensitive to the [OII] $3727 \AA$ emission line of star-burst galaxies at $z=0.35$ and the Ly- $\alpha$ line of young galaxies at $z=3.1$.

[OII] emission line at $z \sim 0.35$ : we do not expect a significant contamination by $[\mathrm{OII}]$ emitters at medium redshifts in the final source catalogs because the emission-line objects have a color excess which implies observed $E W$ s greater than $100 \AA$. Previous studies of [OII] emitters at $z=0.35$ have not found objects with observed $E W$ s greater than $95 \AA$ (Colless et al. 1990; Hammer et al. 1997; Hogg et al. 1998). The long-slit search for low surface brightness Ly- $\alpha$ with fluxes of a few $\times 10^{-18} \mathrm{erg} \mathrm{s}^{-1} \mathrm{~cm}^{-2}$ by Rauch et al. (2008) also reports a low density of [OII] emitters at these faint fluxes.

Ly- $\alpha$ line emitters at $z=3.1$ : on-band/off-band photometric surveys of blank fields provide a non-negligible density of emission objects (Ciardullo et al. 2002b; Gronwall et al. 2007). The 

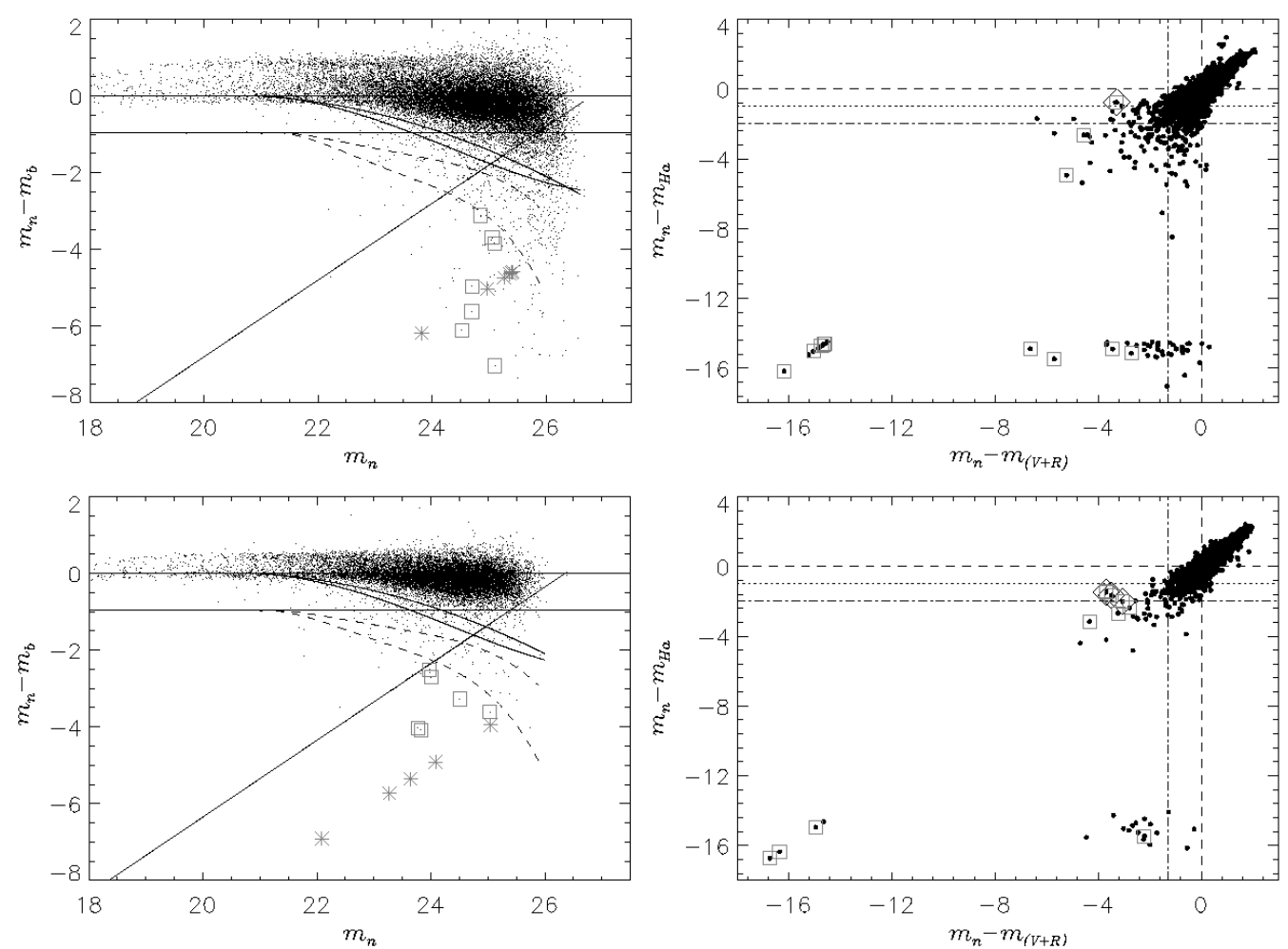

Fig. 4. CMD (left panels) and 2-CD (right panels) of the sources in the SUB2 field (top panels) and SUB3 field (bottom panels) images. On the left: the squares and asterisks represent objects classified as ICPNe with CMDs. On the right: all CMD-identified ICPNe are indicated with open squares; open diamonds show the ICPNe selected from the 2-CD. The continuum and/or H $\alpha$ magnitudes of those objects with no-continuum and/or $\mathrm{H} \alpha$ detections were assigned arbitrarily, as in Fig. 3.

spectroscopic follow-up of these emission line objects showed that they are Ly- $\alpha$ emitters at $z=3.1$ (Gawiser et al. 2007).

In what follows, we adopt a statistical approach to correct for the contribution of the $z=3.1$ Ly- $\alpha$ background objects, by comparing the number density of the "PN-like" objects in the outer Virgo fields with those measured in "blank-fields" (Kudritzki et al. 2000; Ciardullo et al. 2002b; Castro-Rodríguez et al. 2003; Gronwall et al. 2007), normalized by the effective sampled volume.

\subsection{Are ICPNe detected in the Virgo fields outside the cluster core?}

We thus estimate the fraction of background galaxies present in the current catalogs of point-like sources by comparing their LF and number densities with those of Ly- $\alpha$ emitters detected in similar [OIII] on band surveys for objects brighter than $m(5007)=27.0$ and normalized to the effective sampled volumes. We shall compare the different sample for magnitudes brighter than $m(5007)=27.0$; at fainter magnitudes, the automatic classification of pont-like(unresolved) vs. extended(resolved) based on total magnitude vs. small central aperture magnitude plot (see Paper I) becomes unreliable and not uniform among the fields' ICPN catalogs and the comparison blank field catalogs.

Figure 5 compares the LFs of the point-like emission-line objects detected in the LPRX, LPE, LPS, LSF, SUB2 and SUB3 fields with the LF of the Ly- $\alpha$ population from different surveys. We then evaluate the number of expected Ly- $\alpha$ emitters in our field brighter than $m(5007)=27.0$ from the Kudritzki et al. (2000); Ciardullo et al. (2002b); Castro-Rodríguez et al. (2003) surveys, normalized to the same sampled volume. Thus, the expected number of Ly- $\alpha$ galaxies are 65/15/6 in LPRX, 65/15/6 in LPE, 66/16/6 in LPS and 86/20/8 in LSF field.

The blank field survey of Castro-Rodríguez et al. (2003) selected point-like emission line sources with same criteria as the current sample, and it provides a lower inferred number density of Ly- $\alpha$ than either the Ciardullo et al. (2002b) or Kudritzki et al. (2000) surveys. By statistically subtracting the contribution from the background population estimated from Castro-Rodríguez et al. (2003) to the outer Virgo fields, we derive an upper limit to the number of ICPNe. The number of emission-line objects detected in the different fields, and the expected number of Ly- $\alpha$ objects from the Castro-Rodríguez et al. (2003) survey brighter than $m(5007)=27.0$ are 6/6, 14/6, 6/6, 3/8 for LPRX, LPE, LPS, and LSF fields, respectively. The LPE field shows an excess of $8 \pm 4$ emission-line objects over the expected number of background galaxies in this field, while the LSF field shows a lower density than expected, by $5 \pm 2$. We may therefore conclude that the emission-line objects detected in the LPRX, LPS, and LSF fields are compatible with all being Ly- $\alpha$ background galaxies. The emission-line objects detected in LPE are compatible with containing a few ICPNe at the $2 \sigma$ level, as in the SUB2 $/ 3$ fields.

An over-density of emission-line unresolved sources is detected at the RCN1 field position; here we select 28 candidates using the color excess criteria in the CMD, using a deeper offband image than in Paper I, and we expect a population of 8 Ly- $\alpha$ emitters from Castro-Rodríguez et al. (2003) scaled number counts.

On the basis of the previous results, the properties of the unresolved emission line objects in these outer fields are those of the Ly- $\alpha$ population at $z=3.14$. Within our sample, we find that the flux of the brightest emission sources may vary by a factor 2 in different fields, i.e. from 26.0 in LSF field to 26.7 in 

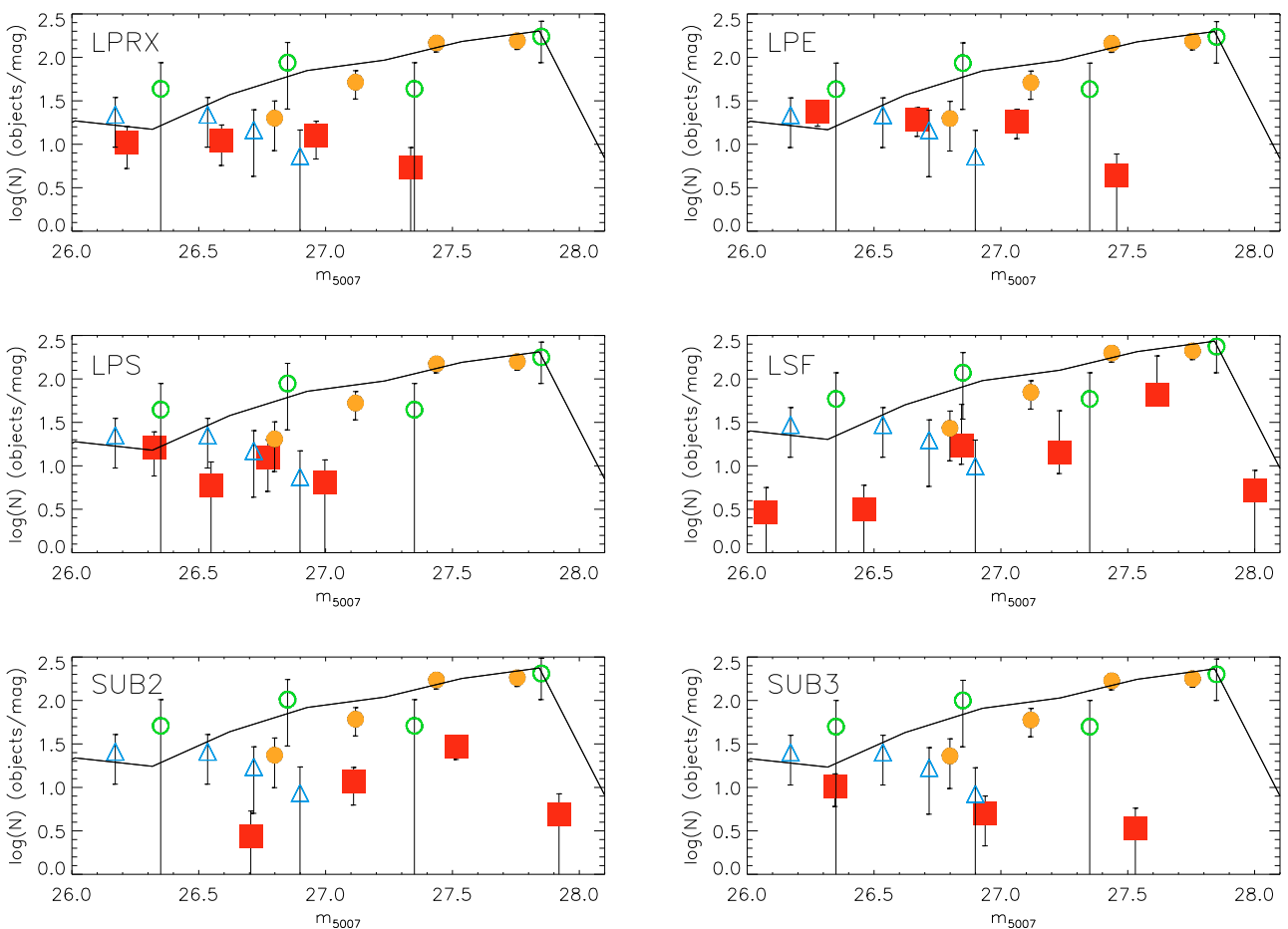

Fig. 5. The luminosity function (LF) of the emission-line objects detected in LPRX, LPE, LPS, LSF, SUB2 and SUB3 is indicated by large full red squares. For comparison, the Ly- $\alpha$ LF from Kudritzki et al. (2000) (open green circles), Ciardullo et al. (2002b) (open blue triangles) and Castro-Rodríguez et al. (2003) (the Leo field; filled orange circles) are also reported in each panel. The continuum line shows the luminosity function of the Ly- $\alpha$ candidates from Gronwall et al. (2007). The LFs of the Ly- $\alpha$ emitters were scaled to the effective surveyed area and wavelength range of the different observed fields.

SUB2 field. The Kudritzki et al. (2000), Ciardullo et al. (2002b), Castro-Rodríguez et al. (2003) and Gronwall et al. (2007) samples show similar field-to-field variation of the Ly- $\alpha$ LF bright end. Since these samples come from surveys that may adopt different selection criteria, we believe that our current results on the field-to-field variations of the Ly- $\alpha$ LF in the six Virgo fields are more robust because they are based on homogeneously selected samples of point-like objects, with deep off-band control images. The variation of the Ly- $\alpha$ properties may be related to the cosmic variance: recent observations by Ouchi et al. (2003) show that Ly- $\alpha$ galaxies are already clustered at $z=4.86$, which implies even greater clustering at $z \approx 3$.

\section{ICPN number density and ICL surface brightness in the Virgo cluster}

On the basis of the statistical subtraction of the contribution from Ly- $\alpha$ emitters at $z=3.14$, we can conclude that:

- in the SUB2 and SUB3 fields, we detected 4 ICPNe in both [OIII] and $\mathrm{H} \alpha$. These are all brighter than $m(5007)=27.0$, i.e. within 0.5 mag of the PNLF bright cut-off at the distance of the Virgo cluster;

- the number densities and LFs of the emission-line objects detected in the outer Virgo fields - LPRX, LPS, and LSF are statistically consistent with those of the population of Ly$\alpha$ background galaxies;

- we detect over-densities in LPE (at the $2 \sigma$ level) and in RCN1 (larger than $3 \sigma$ ) Virgo fields.

On the basis of the number of emission line objects detected in the LPRX, LPS, LSF fields, we can set upper limits to the ICPNe population in each field. We statistically subtract the most conservative number of Ly- $\alpha$ emitters expected in each field based on the Castro-Rodríguez et al. (2003) survey (see discussion in Sect. 4.3), estimate the error-bars from Poisson statistics, and compute the $1 \sigma$ upper limit for the number of ICPNe independently for each field. For LPRX, LPS, and LSF, the expected number of ICPNe brighter than $m(5007)=27.0$ are $0_{-2.4}^{+2.4}, 0_{-2.4}^{+2.4}$, and $-5_{-2.4}^{+2.4}$; for LPE and RCN1 the number of ICPNe is $8_{-2.8}^{+2.8}$ and $20_{-4}^{+4}$ respectively.

\subsection{From ICPN number density to ICL surface brightness}

Since PNe closely follow the distribution of starlight in galaxies (Coccato et al. 2009), the bolometric luminosity of the parent stars of the PN population can be obtained as $L_{\mathrm{bol}}=n_{\mathrm{PN}} / \alpha$, where $n_{\mathrm{PN}}$ is the number of PNs in each field and $\alpha$ is the luminosity-specific PN density. We follow the procedure detailed below:

- the $n_{\mathrm{PN}}$ for a given field is scaled by a factor:

$$
\Delta=\frac{\int_{M^{*}}^{M^{*}+1} \operatorname{PNLF}(m) \mathrm{d} m}{\int_{m^{*}}^{m_{\lim }} \operatorname{PNLF}(m) \mathrm{d} m}
$$

where $\operatorname{PNLF}(m)$ is the analytic expression for the PNLF (Ciardullo et al. 1989), $M^{*}$ and $m^{*}$ denote the absolute and apparent magnitude of its bright cutoff, respectively, and $m_{\mathrm{lim}}$ is the $m(5007)$ limiting magnitude in each field. This scaling ensures that we account for all PNs within 1 mag of $M^{*}$. We then use the corresponding value $\alpha_{1.0}$ to infer the amount of bolometric luminosity in our fields.

- Doherty et al. (2009) computed the $\alpha_{2.5}$ for the halo of M 87 and the ICL directly. The value of $\alpha_{2.5, \mathrm{M} 87}$ is $3.1 \times 10^{-9}$ PN $L_{\odot}^{-1}$; for the ICL $\alpha_{2.5, \text { ICL }}=7.2 \times 10^{-9} \mathrm{PN} L_{\odot}^{-1}$; we refer to 
Table 4. $N_{\mathrm{PN}}$ within $m^{*}+1, B$ band luminosity density and surface brightness values in the Virgo fields. In LPC, LPRX, LPS and LSF we list the upper limits.

\begin{tabular}{cccc}
\hline \hline Field & $N_{\mathrm{PN}}$ & $\begin{array}{c}\Sigma \\
10^{6}\end{array}$ & $\begin{array}{c}L_{\mathrm{B}, \odot} \operatorname{arcmin}^{-2} \\
\mathrm{mag} \mathrm{arcsec}\end{array}$ \\
\hline Core & 17.0 & $3.1 \pm 0.7$ & $29.0 \pm 0.25$ \\
FCJ & 15.0 & $12.2 \pm 3.9$ & $27.5 \pm 0.25$ \\
LPC & $<1.0$ & $<0.2$ & $>32.0$ \\
SUBC & 14.0 & $4.0 \pm 0.7$ & $28.71 \pm 0.3$ \\
\hline LPRX & $<2.4$ & $<0.4$ & $>31.1$ \\
LPE & 8.0 & $1.5 \pm 0.5$ & $29.8 \pm 0.35$ \\
LPS & $<2.4$ & $<0.4$ & $>31.1$ \\
LSF & $<2.4$ & $<0.4$ & $>31.1$ \\
RCN1 & 13.0 & $2.4 \pm 0.6$ & $29.3 \pm 0.3$ \\
SUB2 & 1.0 & $0.2 \pm 0.2$ & $31.8 \pm 1.0$ \\
SUB3 & 3.0 & $0.7 \pm 0.3$ & $30.6 \pm 0.6$ \\
\hline
\end{tabular}

Doherty et al. (2009) for further details on their computation and uncertainties.

- From the analytical expression of the PNLF, we can scale the value of the luminosity-specific PN density at 2.5 mag fainter then the PNLF bright cut off to $1 \mathrm{mag}$ as $\alpha_{1.0}=0.25 \times \alpha_{2.5}$.

- $\alpha$ parameter for the FCJ field - The results of the spectroscopic follow-up by Arnaboldi et al. (2004) and Doherty et al. (2009) show that the halo of M 87 is extended out to about 35 arcmin from the galaxy center. Within that radius, the M 87 halo contributes $70 \%$ of the light as sampled by the number of PNe detected at the position of the FCJ field. For this field position, we use a value of $\alpha_{1.0}=1.1 \times 10^{-9} \mathrm{PN} L_{\odot}^{-1}$ which is the average value of $\alpha_{1.0, \mathrm{M} 87}$ and $\alpha_{1.0, \mathrm{ICL}}$, weighted by the fractions of PNs that are bound to M 87 (70\%) and those unbound (30\%). Because the FCJ field is at a distance of about 14 arcmin from the center of M 87, we can check the derived surface brightness value at this field position with the $V$ band surface brightness measurements of Kormendy et al. (2009).

- $\alpha$ parameter for the SUBC field - we use $\alpha_{1.0}=1.3 \times$ $10^{-9} \mathrm{PN} L_{\odot}^{-1}$, which is the average of $\alpha_{1.0, \mathrm{M} 87}$ and $\alpha_{1.0, \text { ICL }}$. Here we assume that M 84 has the same $\alpha_{1.0}$ value as M 87 and the fractions of bound and unbound PNs in this photometric sample is $50 \%$ each.

- $\alpha$ parameter for the outer fields - For the fields at larger distances, we use $\alpha_{1.0, \mathrm{ICL}}=4.0 \times 10^{-9} \mathrm{PN} L_{\odot}^{-1}$.

- Errors on the inferred luminosities and surface brightness are estimated from Poisson statistics.

We compute the bolometric luminosities at each field position from the number of observed ICPNe, or their upper limit, and the $\alpha$ parameter. Using the bolometric correction to the $B$ band (Buzzoni et al. 2006) and $(B-V)$ colors, we derive the $B$ band luminosities and surface brightnesses. The adopted $B-V$ colors are 0.9 for the fields at large distances, and $(B-V)=1.02$ for those fields near either M 87 and M 84. In Table 4, for each field we list the number of PNe within $m^{*}+1, N_{\mathrm{PN}}$, the resulting mean values of the $B$-band luminosity, $\Sigma$, and surface brightness $\mu_{\mathrm{B}}$.

\subsection{ICL measurements in the Virgo cluster from previous work: the Feldmeier (20004b) sample}

Several regions in the Virgo core and around M 49 were surveyed by Feldmeier et al. (1998, 2003, 2004a) for ICPNe, covering a total area of $0.89 \mathrm{deg}^{2}$. The positions of these fields are shown in Fig. 1, and a summary of their $\mu_{V}$ measurements is provided in Table 5. They find an excess of emission line sources in all fields,
Table 5. Surface brightness measurements in the Feldmeier et al. (2004a) fields.

\begin{tabular}{cccc}
\hline \hline Field & $\begin{array}{c}\mu_{\text {ICL,V }} \\
\mathrm{mag} \mathrm{arcsec}^{-2}\end{array}$ & $\begin{array}{c}\mu_{\text {ICL,B }} \\
\mathrm{mag} \mathrm{arcsec}^{-2}\end{array}$ & $B-V$ \\
\hline F04-2 & 27.4 & 28.3 & 0.96 \\
F04-3 & 26.5 & 27.5 & 1.02 \\
F04-4 & 27.4 & 28.4 & 1.02 \\
F04-5 & 27.3 & 28.3 & 1.02 \\
F04-6 & 28.1 & 29.1 & 0.96 \\
F04-7 & 28.4 & 29.4 & 1.02 \\
F04-8 & - & - & - \\
\hline
\end{tabular}

at about 5 times the density of line emitters in blank fields, but for Field F04-8, where the emission-line object number density is consistent with the galaxy background population. To compare these measurements with ours, we take their $\mu_{v}$ and add a $(B-$ $V$ ) color term determined from the color of the continuum light of the nearest bright galaxy; the computed values are shown in Col. \#4, in Table 5.

The field with the brightest surface brightness measurement is F04-3 (FCJ in this work; see also Paper I) at $60 \mathrm{kpc}$ from the center of M 87. This field is within the M 87 halo, as shown by the deep photometry of the Virgo core by Mihos et al. (2005) and the PN kinematics (Arnaboldi et al. 2004). The $\mu_{\mathrm{B}}$ measurement for F04-3 agrees with the independently derived $\mu_{\mathrm{B}}$ for FCJ in Table 4 and with the surface brightness profile of M 87 measured by Kormendy et al. (2009) at the FCJ distance from the center of M 87. The spectroscopic follow-up indicates that the M 87 halo contributes $70 \%$ of the light at this field position, and $30 \%$ of the sample is made up of free-floating stars, i.e. the true ICL component. The surface brightness of the ICL at the position of the FCJ field is then $\mu_{\mathrm{B}}=28.8 \mathrm{mag} \operatorname{arcsec}^{-2}$.

\subsection{How well do ICPNe follow light}

Mihos et al. (2009) investigated the correlation between the ICPN distribution and the diffuse light in the Virgo core (Mihos et al. 2005). Their results indicate that there is a correspondence on large scales $(\sim 100 \mathrm{kpc})$, which is more robust when the ICPN catalogs have a lower fraction of background contaminants, as in the case of the SUBC field (Paper II and Okamura et al. 2002), or in the higher surface brightness regions within the extended galaxy halos. A possible source of scatter is also the dependence of the luminosity-specific PN parameter on stellar populations (Ciardullo et al. 2005; Buzzoni et al. 2006).

The procedure adopted in this work maps the over-density of point-like emitters with respect to a statistically averaged background population, in fields whose area is about $150 \times 150 \mathrm{kpc}^{2}$. As described in Sect. 5.1, the number of PNe from each overdensity is mapped into a bolometric luminosity according to the most appropriate luminosity-specific PN density parameter value for that field. Our comparison with the Mihos et al. (2009) results indicate that the luminosities computed in this work are robust with respect to the variety of effects that can act to wash out the correlation between PN number density distribution and light.

\section{Discussion}

\subsection{The diffuse light in the Virgo cluster}

Several fields outside the Virgo core region contain a population of emission sources consistent with background Ly- $\alpha$ objects. 
Clear over-density of emission line objects of at least a factor of 5 with respect to the averaged Ly- $\alpha$ emitters are detected in the Virgo core region, around M 49 (Feldmeier et al. 2003, 2004a), and in RCN1 and LPE, at a lower $\sigma$ level.

The ICL and the extended halos from bright galaxies - The diffuse light observed in the core of a galaxy cluster contains several luminous stellar components that add up along the lineof-sight (LOS) to the cluster center: the extended faint halos of the brightest galaxies and the ICL contribution, defined as the light coming from stars not bound to individual galaxies. When computing the ICL fraction in the Virgo core, the surface brightness measurements must be corrected for the fraction of stars bound to the extended halos of individual galaxies. The results from Arnaboldi et al. (2004) and Doherty et al. (2009) on the LOS velocity distribution of the PNe indicate that the halos of M 84, M 86 and M 87 are extended, out to $150 \mathrm{kpc}$ in the case of M 87.

When we select only true ICPNe, we measure a surface brightness for the ICL of about $\mu_{\mathrm{B}}=28.8 \mathrm{mag} \mathrm{arcsec}^{-2}$ in FCJ and 29.0-29.5 in Core/SUBC. These surface brightness values are similar to those inferred from the detection of IC RGB stars (Ferguson et al. 1998; Durrell et al. 2002; Williams et al. 2007).

The results from the spectroscopic follow-up imply that the measurements of the ICL based on a surface brightness threshold, e.g. $\mu_{v}>26.5$, will lead to an overestimate of the ICL, since it includes the light contributions from extended galaxy halos.

\subsection{On the elongated distribution of the ICL}

The Feldmeier et al. (2004a)'s "smooth" elongated distribution for the ICL, of about $4 \mathrm{Mpc}$ along the LOS to the Virgo core, was derived from the following observational results:

- the relative brighter cut-off of the IC PNLF in sub-clump A, around M 87, vs. sub-clump B, around M 49, placing the subclump $\mathrm{A}$ at $4 \mathrm{Mpc}$ in front of sub-clump B;

- same number density of ICPNe in these fields.

The evidence for a substantial depth along the LOS is weakened by the results that some brightening of the PNLF in the M 87 halo may be intrinsic (Arnaboldi et al. 2008). The presence of an extended halo around M49, which is to be expected given the results for M 87 (Doherty et al. 2009), may explain the same number density of PNe around M49 as in M87, because the M 49 halo reaches similar surface brightness as the M 87 one at these fields' relative distances from the galaxy center.

The upper limits in the LPC, LPS and LPRX fields from this work, and no-PN detection in F04-8 (Feldmeier et al. 2003) show that there is not a uniform distribution of ICL all across the region from sub-clump A-M 87 to subclump B-M 49 (Binggeli et al. 1987). The ICL drops below the detection limit in the region of the sky between these two Virgo sub-clumps. The current results indicate that the ICL is mostly associated with, and confined to, these high density regions.

\subsection{The ICL surface brightness radial profile}

In Fig. 6 we now plot the surface brightness values from Tables 4 and 5 as a function of the field distances from M 87, for an easier comparison with the Mihos et al. (2005) and Kormendy et al. (2009) photometry.

Properties of the ICL distribution in the Virgo cluster - the ICPN survey in the Virgo cluster indicates that the ICL is not homogeneously distributed in the core and at larger cluster radii. In

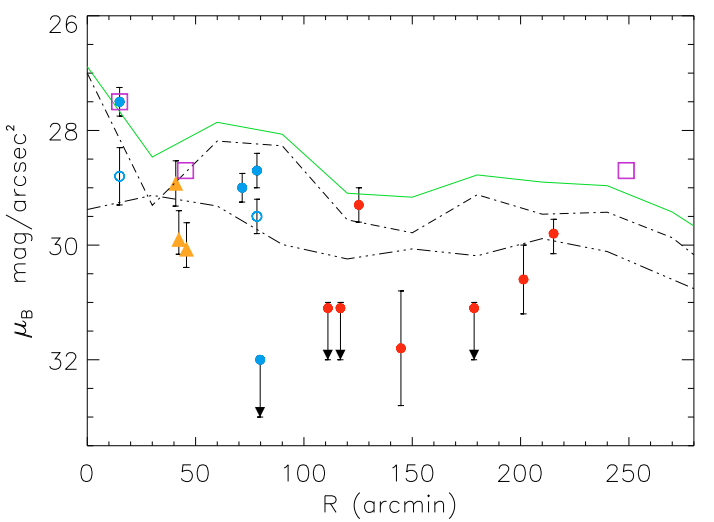

Fig. 6. Surface brightness measurement of diffuse light in the Virgo fields (points) compared with the surface brightness profile of the Virgo galaxies averaged in annuli (lines); radial distances are computed with respect to M 87. The green line represents the radial surface brightness profile from light in Virgo galaxies from Binggeli et al. (1987). The dotted-dashed and double dotted-dashed lines correspond to the surface brightness profile associated with giants and dwarf galaxies, respectively. The full blue dots show the surface brightness measurements in the FCJ, Core, SUBC and LPC fields. The open circles at the FCJ, SUBC field position indicates the ICL surface brightness computed from the ICPNe not bound to galaxy halos. The triangles represent the surface brightness of the ICL based on IC RGB star counts (Ferguson et al. 1998; Durrell et al. 2002; Williams et al. 2007). The full red dots show the surface brightness measurements in the RCN1, LPE, SUB2, and SUB3 fields. The arrows indicate the upper limits for the measurements in LPRX, LPS and LSF. The magenta open squares indicate the surface brightness average values $\mu_{\mathrm{B}}$ at 15,50 and 240 arcmin computed from the measurements listed in Table 5, from Feldmeier et al. (2004a) data; the measurements at $240 \mathrm{arcmin}$ (F04-2 and F04-6) are close to M 49, see Fig. 1. Radial distances are computed with respect to M 87.

the core, we have an upper limit for the LPC field, and in Field F04-8 (Feldmeier et al. 2003, 2004a). Furthermore the deep image of the central region of the Virgo cluster shows the presence of complex structures at all scales (Mihos et al. 2005). Both results indicate that the distribution of the diffuse light in the central region is inhomogeneous, further supporting the evidence that the core is not relaxed, see Binggeli et al. (1993), Paper III, and Doherty et al. (2009).

The comprehensive summary of all surface brightness measurements in Fig. 6 indicates that most of the diffuse light is detected in fields located in the core, within a distance of about 80 arcmin from M 87. Outside this region, the mean surface brightness decreases sharply, and the ICL is present in isolated pointings, e.g. RCN1 and LPE.

Diffuse stellar light is also measured in "sub-structures", around M 49 and in the M 60/M 59 sub-group. The fields F04-2 and F04-6 from Feldmeier et al. (2004a) are situated in the outer regions of M 49, at about $150 \mathrm{kpc}$ from the galaxy center. These fields may contain PNe from the halo of M 49 and the ICL component, which may have formed within sub-clump B of the Virgo cluster (Binggeli et al. 1987). Because the spectroscopic followup in not yet available for these PN candidates, we cannot quantify the fraction of light in the M 49 halo and ICL for these fields.

Using a technique similar to Durrell et al. (2002) and Williams et al. (2007), Yan et al. (2008) serendipitously detected an excess of point-like objects in two ACS fields from M 60 at 7 and 15 arcmin. The number density of the excess field population shows a decreasing gradient with increasing distance from M 60. This population starts at $m_{i 775}=26.2$ and the average surface brightness is $26.6 \mathrm{mag} \operatorname{arcsec}^{-2}$ in the $I$ band in the field at 7 arcmin from M60. These values are very similar to those 
measured by the Durrell et al. (2002) observations of the IC RGB in the Virgo core. Yan et al. (2008) argue that they detected a stellar population associated with the M 60/M 59 pair. This result may indicate that the M60/M59 sub-group is yet another region of the Virgo cluster where the intra-group-light and diffuse halos are being assembled.

The current result on the slope of the radial profile of the ICL is somewhat different from the slow decline in ICPN number density described by Feldmeier et al. (2004a). A steeper radial ICPN density profile is now derived because the current survey probes areas between the sub-clump A and sub-clump B in the Virgo cluster, where only upper limits are set.

\subsection{Fraction of the ICL light vs. galaxy light in the Virgo core}

When we average the measurements of the ICL in the core region defined as the circular area of $80 \operatorname{arcmin}$ radius ${ }^{4}$ centred on the Virgo cluster center given by Binggeli et al. (1987), we obtain a measurement of $\mu_{\mathrm{B}}=29.3 \mathrm{mag} \mathrm{arcsec}^{-2}$. The ICL luminosity in this area is $L_{\odot, \mathrm{ICL}}=39.2 \times 10^{9} L_{\odot, \mathrm{B}}$. From Binggeli et al. (1987) the cumulative luminosity from all galaxy types within $1.3 \mathrm{deg}=80 \mathrm{arcmin}$ radius of the Virgo cluster center is $L_{\odot, \text { gal }}=58.16 \times 10^{10} L_{\odot, \mathrm{B}}$. Our best estimate of the fraction of ICL, defined as the un-bound stellar component in the Virgo cluster core, amounts to $\sim 7 \%$ of the total light in Virgo cluster galaxies.

The current fraction is lower than the recent measurement from Feldmeier et al. (2004a), using a very similar technique. This is not unexpected: we have corrected the surface brightness measurements in the inner Virgo core fields by subtracting a significant fraction of PN bound to bright galaxy halos, which is between $70 \%$ and $50 \%$, of the total $N_{\text {PN }}$ measured in those fields. We think that the current (conservative) ICL fraction is a robust estimate of the light from true-unbound stars within $80 \mathrm{arcmin}$ centred on M 87.

\subsection{Implications for the formation of the ICL in the Virgo cluster}

We can summarize the current results as follows:

- the ICL in Virgo is concentrated in the core, with $\mu_{\mathrm{ICL}, \mathrm{B}}$ in the range $28.8-30 \mathrm{mag} \operatorname{arcsec}^{-2}$;

- in several fields at $R>80^{\prime}=340 \mathrm{kpc}$ we can set only upper limits to the ICL surface brightness;

- at $R>80^{\prime}$, the ICL signal is confined to isolated fields (RCN1,LPE);

- diffuse stellar light defined as extended halo plus ICL is present in other Virgo sub-structures (central region of subclump B, M 60/M 59 sub-group).

The distribution of the ICL in the core and at larger scales in the Virgo cluster shows that the ICL is more centrally concentrated than the galaxies, see Fig. 6 . This result is consistent with the measurements of Zibetti et al. (2005) and the predictions of Murante et al. (2004, 2007).

The ICL distribution shows a high degree of substructures both in the Virgo core and at larger radii. This result is supported by deep surface brightness photometry (Mihos et al. 2005), and ICPN number counts. The presence of sub-structures is predicted by cosmological simulations of structure formation

\footnotetext{
4 Because this circular area includes regions with only upper limits, our average surface brightness is lower than that measured by Mihos et al. (2005) in the region between M 87 and M 86.
}

(Napolitano et al. 2003; Sommer-Larsen et al. 2005; Murante et al. 2007). The detection of extended halos and intracluster/intra-group light in Virgo sub-structures supports a hierarchical formation mechanism for the ICL as described by Rudick et al. (2006): we are observing the ICL component in sub-structures, which may then become completely unbound as these sub-structures fall into the densest part of the cluster.

Current data are consistent with most ICL being associated with the formation history of the brightest cluster galaxies, which explains the central concentration of the ICL in the core and to sub-structures. Tidal effects are indeed at work at larger distances from the center and they can generate some ICL. The ICPNe detected in RCN1, LPE and SUB2/3 would likely come from such processes, but the amount of light detected in these fields is small compared with that sampled close to the big ellipticals, as supported by the observational results of this work.

\section{The local mean luminous density}

From the lack of PN detections in several of our fields in the Virgo cluster, we can derive a limit on the luminosity density in an assumed homogeneous distribution of stars in the local volume. Fukugita et al. (1998) compiled measurements of the mean luminous density contributed by high surface brightness galaxies at moderately low redshift, and adopted $\epsilon_{0}=$ $2 \times 10^{8} h L_{\mathrm{B}, \odot} \mathrm{Mpc}^{-3}$. In what follows, we make the assumption that the mean luminous density is distributed uniformly in the local volume and compute the number of PNe associated with this diffuse component in the surveyed area of the Virgo outer fields and at the [OIII] limiting magnitude $m_{\lim }(5007)=27.0$.

Each volume element along the LOS to the Virgo fields contributes the following number of $\mathrm{PNe}$

$\mathrm{d} N_{\mathrm{PN}}(r)=\alpha(r) \times \mathrm{d} L(r)$

where $L(r)$ is the luminosity associated with the volumetric luminous density

$\mathrm{d} L(r)=\epsilon_{0} \times S \times \mathrm{d} r=\frac{\epsilon_{0} \Omega}{206265^{2}} r^{2} \mathrm{~d} r$

$\epsilon_{0}=2 \times 10^{8} h L_{\mathrm{B}, \odot} \mathrm{Mpc}^{-3}$ (see Fukugita et al. 1998), $\Omega$ is the area in $\operatorname{arcsec}^{2}, \alpha(r)$ is the luminosity-specific planetary nebula number and predicts the number of PNe associated with the total luminosity $L(r)$ at radial distance $r$. Its value depends on the relative depth of the on-band survey $\left(m_{\mathrm{lim}}\right)$ with respect to the faint limit of the PNLF, e.g. the apparent magnitude of $M^{*}+8$ at the distance $r$.

The total number of PNe associated with the volumetric density is then given by the integral of Eq. (2)

$$
\begin{aligned}
N_{\mathrm{PN}, \text { tot }} & =\int_{0}^{r_{\max }} N_{\mathrm{PN}}(r) \mathrm{d} r=\int_{0}^{r_{\max }} \alpha(r) L_{T}(r) \mathrm{d} r \\
& =\frac{\epsilon_{0} \Omega}{206265^{2}} \int_{0}^{r_{\max }} \alpha(r) r^{2} \mathrm{~d} r
\end{aligned}
$$

and $r_{\max }$ is set by the survey depth, and it is the distance at which the apparent magnitude of the PNLF bright cut-off $m^{*}$ is equal to $m_{\mathrm{lim}}$.

The luminosity-specific planetary nebula number $\alpha-$ the PN population $\left(N_{\mathrm{PN}}\right)$ associated with a stellar population with luminosity $L_{T}$ is :

$$
\begin{aligned}
N_{\mathrm{PN}} & =\int_{M^{*}}^{M^{*}+8} \operatorname{PNLF}(M) \mathrm{d} M \\
& =\int_{M^{*}}^{M^{*}+8} k \mathrm{e}^{0.307 M}\left(1.0-\mathrm{e}^{\left(3 *\left(M^{*}-M\right)\right)}\right) \mathrm{d} M=\alpha L_{T}
\end{aligned}
$$


where $M^{*}=-4.5$ is the absolute magnitude of the bright cut-off of the PNLF, and $\alpha$ is the luminosity-specific PN number. $\alpha$ is given by Buzzoni et al. (2006) for stellar populations with different ages and metallicities. The maximum theoretical value of $\alpha$ which gives the largest PN population for a given luminosity is $\alpha_{\max }=1 \mathrm{PN} \times\left(1.85 \times 10^{6} L_{\odot}\right)^{-1}$. From Eq. (2) we see that the PNLF extends over 8 mag, but one sees smaller magnitude ranges as distance increases. The $\alpha$ parameter must then be corrected using the double-exponential PNLF analytical formula in Eq. (5) for the appropriate faint cut-off magnitude set by the the minimum between $m_{\mathrm{lim}}$ and the apparent magnitude corresponding to $M^{*}+8$ at distance $r$.

Predicted number of PNe - we use Eq. (4) to compute the number of PNe we would predict if the Fukugita et al. (1998) density was homogeneously distributed. The number of PNe that we would detect by surveying $1.75 \mathrm{deg}^{2}$ (total area covered by the $L *$ fields plus SUB2 and SUB3 fields) down to $m_{\mathrm{lim}}=27.0$, is $N_{\mathrm{PN} \text {,tot }}=4.6$ with an expected $m(5007)$ magnitude distribution that peaks at $\sim 26.0$. We conclude that we cannot set limits on the homogeneously distributed luminous density in the local universe, and surveys of several hundred square degrees are required to detect a statistically significant numbers of the brighter PNe.

\section{Conclusions}

We have surveyed several regions of the Virgo cluster located at different distances from $\mathrm{M} 87$, covering a total area of 3.3 square degrees. These outer fields in the Virgo cluster were imaged through off- and on-band filters designed for detecting PNe at the distance of the Virgo cluster. The result of this survey provides a homogeneous large scale study of the diffuse stellar light in Virgo.

The results from the current study indicate that the diffuse stellar component is present in the central region of the cluster, within a radial distance of 80 arcmin from the cluster center. The intracluster light in the core has a surface brightness of $\mu_{\mathrm{B}}=$ 28.8-30 mag arcsec ${ }^{-2}$, and on average it amounts to $\sim 7 \%$ of the total galaxy light in this region. The distribution of the ICL in the cluster central region is not homogeneous, with areas where only upper limits on the number of PNe can be set. This nonuniformity indicates that the ICL is not relaxed in the core.

At distances of more than 80 arcmin from M 87, the surface brightness profile of the diffuse light has a sharp cut-off of about $2 \mathrm{mag}$. At these larger radii the detection of ICL is confined in single fields, e.g. RCN1, LPE and SUB2/3, or is associated with local substructures, e.g. sub-clump B, the M 60/M 59 sub-group. The observations indicate that the ICL in Virgo is more centrally concentrated than cluster galaxies, and does not support a homogeneous elongated distribution of the ICL similar to late-type Virgo galaxies (Yasuda et al. 1997; Solanes et al. 2002).

The concentration of the ICL toward the cluster high density regions links the formation of the ICL with the formation and evolution of the most luminous cluster galaxies (Rudick et al. 2006; Murante et al. 2007).

Acknowledgements. The authors would like to thank the anonymous referee for the careful reading of the manuscript and the extensive report. J.A.L.A. acknowledges the travel support from ESO Director Discretionary Funds 2008 during the writing of this manuscript. This article is based on observations made with 1) the Isaac Newton Telescope operated on the island of La Palma by the Isaac Newton Group in the Spanish del Roque de los Muchachos Observatory; 2) the ESO/MPI $2.2 \mathrm{~m}$ telescope at La Silla (Chile); and 3) the Subaru Telescope, which is operated by the National Astronomical Observatory of Japan. NCR and JALA were founded by the grant AYA2007-67965-C03-01.

\section{Appendix A: Catalog of the point-like emission line objects detected in the current survey}

Table A.1. Emission-line objects detected in the LPRX field.

\begin{tabular}{cccc}
\hline \hline Name & $\alpha(J 2000)$ & $\delta(J 2000)$ & $m(5007)$ \\
\hline RCN1-1 & $12: 25: 49.69$ & $14: 22: 08.86$ & 25.478 \\
RCN1-2 & $12: 27: 11.53$ & $14: 06: 36.37$ & 25.913 \\
RCN1-3 & $12: 27: 04.63$ & $14: 14: 55.15$ & 25.915 \\
RCN1-4 & $12: 26: 01.43$ & $14: 19: 13.80$ & 25.926 \\
RCN1-5 & $12: 26: 11.00$ & $14: 08: 49.73$ & 25.926 \\
RCN1-6 & $12: 26: 30.36$ & $14: 07: 31.63$ & 26.194 \\
RCN1-7 & $12: 26: 40.40$ & $14: 08: 35.24$ & 26.240 \\
RCN1-8 & $12: 27: 12.32$ & $14: 01: 27.14$ & 26.249 \\
RCN1-9 & $12: 26: 30.01$ & $14: 17: 09.80$ & 26.295 \\
RCN1-10 & $12: 25: 34.38$ & $13: 58: 09.08$ & 26.297 \\
RCN1-11 & $12: 26: 05.27$ & $14: 11: 14.95$ & 26.311 \\
RCN1-12 & $12: 25: 35.01$ & $13: 59: 42.98$ & 26.312 \\
RCN1-13 & $12: 27: 13.55$ & $14: 17: 48.79$ & 26.343 \\
RCN1-14 & $12: 26: 59.59$ & $14: 09: 56.40$ & 26.348 \\
RCN1-15 & $12: 27: 08.51$ & $14: 08: 29.50$ & 26.355 \\
RCN1-16 & $12: 26: 59.25$ & $13: 53: 42.86$ & 26.359 \\
RCN1-17 & $12: 26: 28.77$ & $14: 08: 34.98$ & 26.394 \\
RCN1-18 & $12: 26: 33.17$ & $14: 16: 20.35$ & 26.489 \\
RCN1-19 & $12: 26: 28.15$ & $14: 08: 04.62$ & 26.490 \\
RCN1-20 & $12: 25: 55.82$ & $14: 09: 08.78$ & 26.572 \\
RCN1-21 & $12: 27: 04.90$ & $14: 10: 43.36$ & 26.602 \\
RCN1-22 & $12: 25: 54.25$ & $13: 55: 17.77$ & 26.657 \\
RCN1-23 & $12: 27: 15.57$ & $14: 23: 12.65$ & 26.674 \\
RCN1-24 & $12: 27: 07.64$ & $14: 16: 10.91$ & 26.681 \\
RCN1-25 & $12: 25: 27.23$ & $14: 11: 33.21$ & 26.682 \\
RCN1-26 & $12: 27: 07.01$ & $13: 59: 56.88$ & 26.686 \\
RCN1-27 & $12: 26: 34.64$ & $14: 11: 55.75$ & 26.689 \\
RCN1-28 & $12: 26: 09.25$ & $13: 57: 22.98$ & 26.688 \\
\hline & & &
\end{tabular}

Table A.2. Emission-line objects detected in the LPRX field.

\begin{tabular}{cccc}
\hline \hline Name & $\alpha(\mathrm{J} 2000)$ & $\delta(\mathrm{J} 2000)$ & $m(5007)$ \\
\hline LPRX-1 & $12: 27: 20.377$ & $9: 17: 37.14$ & 26.03 \\
LPRX-2 & $12: 26: 32.996$ & $9: 23: 48.76$ & 26.42 \\
LPRX-3 & $12: 28: 11.142$ & $9: 25: 14.66$ & 26.47 \\
LPRX-4 & $12: 27: 25.608$ & $9: 16: 14.40$ & 26.53 \\
LPRX-5 & $12: 27: 48.012$ & $9: 14: 26.81$ & 26.78 \\
LPRX-6 & $12: 26: 42.855$ & $9: 16: 12.58$ & 26.94 \\
LPRX-7 & $12: 27: 25.148$ & $9: 12: 53.31$ & 27.19 \\
LPRX-8 & $12: 27: 31.843$ & $9: 37: 10.15$ & 27.33 \\
LPRX-9 & $12: 26: 40.641$ & $9: 28: 57.08$ & 27.36 \\
\hline
\end{tabular}

Table A.3. Emission-line objects detected in the LPS field.

\begin{tabular}{cccc}
\hline \hline Name & $\alpha(\mathrm{J} 2000)$ & $\delta(\mathrm{J} 2000)$ & $m(5007)$ \\
\hline LPS-1 & $12: 27: 25.204$ & $10: 51: 38.34$ & 26.21 \\
LPS-2 & $12: 26: 38.216$ & $10: 35: 49.34$ & 26.36 \\
LPS-3 & $12: 27: 34.998$ & $10: 26: 46.14$ & 26.38 \\
LPS-4 & $12: 27: 10.849$ & $10: 43: 44.31$ & 26.75 \\
LPS-5 & $12: 27: 33.526$ & $10: 44: 30.01$ & 26.98 \\
LPS-6 & $12: 25: 59.211$ & $10: 44: 33.17$ & 27.01 \\
LPS-7 & $12: 27: 38.171$ & $10: 53: 50.34$ & 27.10 \\
\hline
\end{tabular}


Table A.4. Emission-line objects in the LPE field.

\begin{tabular}{cccc}
\hline \hline Name & $\alpha(\mathrm{J} 2000)$ & $\delta(\mathrm{J} 2000)$ & $m(5007)$ \\
\hline LPE-1 & $12: 17: 58.471$ & $13: 40: 07.24$ & 26.08 \\
LPE-2 & $12: 17: 32.446$ & $13: 48: 31.87$ & 26.15 \\
LPE-3 & $12: 17: 39.261$ & $13: 32: 27.24$ & 26.19 \\
LPE-4 & $12: 18: 04.059$ & $13: 43: 38.15$ & 26.19 \\
LPE-5 & $12: 17: 59.871$ & $13: 43: 18.95$ & 26.40 \\
LPE-6 & $12: 17: 17.932$ & $13: 28: 25.66$ & 26.43 \\
LPE-7 & $12: 16: 04.795$ & $13: 40: 59.73$ & 26.44 \\
LPE-8 & $12: 16: 36.738$ & $13: 45: 45.07$ & 26.52 \\
LPE-9 & $12: 16: 02.328$ & $13: 31: 03.16$ & 26.71 \\
LPE-10 & $12: 17: 01.922$ & $13: 56: 48.82$ & 26.8 \\
LPE-11 & $12: 18: 04.406$ & $13: 43: 05.79$ & 26.8 \\
LPE-12 & $12: 16: 54.828$ & $13: 58: 22.60$ & 26.9 \\
LPE-13 & $12: 18: 14.529$ & $13: 39: 13.96$ & 27.0 \\
LPE-14 & $12: 18: 07.388$ & $13: 52: 02.52$ & 27.0 \\
LPE-15 & $12: 16: 53.685$ & $13: 29: 48.63$ & 27.1 \\
LPE-16 & $12: 16: 48.095$ & $13: 52: 14.56$ & 27.2 \\
LPE-17 & $12: 16: 59.375$ & $13: 44: 38.69$ & 27.3 \\
LPE-18 & $12: 16: 41.977$ & $13: 34: 25.83$ & 27.3 \\
LPE-19 & $12: 18: 09.217$ & $13: 50: 04.31$ & 27.4 \\
LPE-20 & $12: 18: 11.198$ & $13: 57: 59.73$ & 27.6 \\
\hline
\end{tabular}

Table A.5. Emission-line objects detected in the LSF field.

\begin{tabular}{cccc}
\hline \hline Name & $\alpha(\mathrm{J} 2000)$ & $\delta(\mathrm{J} 2000)$ & $m(5007)$ \\
\hline LSF-1 & $12: 37: 42.215$ & $12: 15: 43.10$ & 25.99 \\
LSF-2 & $12: 39: 13.235$ & $12: 14: 38.27$ & 26.83 \\
LSF-3 & $12: 38: 07.374$ & $12: 07: 34.85$ & 27.08 \\
LSF-4 & $12: 39: 13.669$ & $12: 01: 46.78$ & 27.17 \\
LSF-5 & $12: 37: 42.473$ & $12: 11: 20.97$ & 27.35 \\
LSF-6 & $12: 39: 20.662$ & $12: 16: 37.00$ & 27.36 \\
LSF-7 & $12: 39: 09.730$ & $12: 10: 50.76$ & 27.37 \\
LSF-8 & $12: 39: 13.045$ & $12: 11: 06.79$ & 27.66 \\
LSF-9 & $12: 37: 46.282$ & $11: 55: 14.22$ & 27.70 \\
LSF-10 & $12: 38: 47.034$ & $12: 18: 00.90$ & 27.70 \\
LSF-11 & $12: 37: 28.577$ & $12: 17: 48.96$ & 27.80 \\
LSF-12 & $12: 37: 46.895$ & $12: 15: 24.38$ & 27.91 \\
LSF-13 & $12: 37: 38.274$ & $12: 20: 31.31$ & 27.93 \\
LSF-14 & $12: 39: 15.965$ & $11: 55: 43.51$ & 27.93 \\
LSF-15 & $12: 37: 30.846$ & $12: 19: 05.18$ & 27.97 \\
LSF-16 & $12: 39: 27.344$ & $12: 20: 25.23$ & 27.99 \\
LSF-17 & $12: 39: 20.736$ & $12: 05: 44.44$ & 28.02 \\
LSF-18 & $12: 37: 29.078$ & $12: 22: 37.17$ & 28.03 \\
LSF-19 & $12: 38: 05.800$ & $12: 01: 34.96$ & 28.03 \\
LSF-20 & $12: 38: 41.080$ & $11: 57: 07.98$ & 28.04 \\
LSF-21 & $12: 38: 00.317$ & $12: 21: 35.34$ & 28.06 \\
LSF-22 & $12: 38: 37.500$ & $12: 05: 56.12$ & 28.09 \\
LSF-23 & $12: 39: 23.081$ & $12: 12: 33.49$ & 28.15 \\
\hline
\end{tabular}

Table A.6. Emission-line objects detected in the SUB2 field.

\begin{tabular}{cccccc}
\hline \hline Name & $\alpha(\mathbf{J} 2000)$ & $\delta(\mathbf{J} 2000)$ & $m(5007)$ & $m_{\mathrm{H} \alpha}$ & $m_{(V+R)}$ \\
\hline SUB2-1 & $12: 23: 22.524$ & $14: 03: 21.68$ & 26.30 & - & - \\
SUB2-2 & $12: 24: 23.113$ & $14: 20: 50.64$ & 27.01 & - & 30.22 \\
SUB2-3 & $12: 24: 45.272$ & $14: 18: 52.58$ & 27.18 & - & 29.91 \\
SUB2-4 & $12: 24: 35.834$ & $14: 05: 36.93$ & 27.19 & - & 29.26 \\
SUB2-5 & $12: 24: 45.103$ & $14: 16: 12.88$ & 27.34 & - & 27.56 \\
SUB2-6 & $12: 24: 46.116$ & $14: 13: 04.52$ & 27.46 & - & - \\
SUB2-7* & $12: 24: 15.861$ & $14: 04: 17.51$ & 27.54 & 25.81 & 28.33 \\
SUB2-8 & $12: 25: 01.022$ & $14: 02: 10.35$ & 27.58 & - & 28.53 \\
SUB2-9 & $12: 23: 39.559$ & $14: 18: 15.09$ & 27.59 & - & 31.73 \\
SUB2-10 & $12: 24: 35.553$ & $13: 55: 21.40$ & 27.75 & - & - \\
SUB2-11 & $12: 23: 59.558$ & $14: 13: 02.89$ & 27.85 & - & - \\
SUB2-12 & $12: 23: 04.010$ & $14: 06: 12.86$ & 27.88 & - & - \\
SUB2-13 & $12: 23: 25.975$ & $14: 05: 48.30$ & 27.90 & - & - \\
\hline
\end{tabular}

Objects with $(*)$ are ICPNe.
Table A.7. Emission-line objects detected in the SUB3 field.

\begin{tabular}{cccccc}
\hline \hline Name & $\alpha(\mathrm{J} 2000)$ & $\delta(\mathrm{J} 2000)$ & $m(5007)$ & $m_{\mathrm{H} \alpha}$ & $m_{(V+R)}$ \\
\hline SUB3-1 & $12: 24: 21.128$ & $15: 32: 10.67$ & 24.57 & - & - \\
SUB3-2 & $12: 25: 02.814$ & $15: 16: 42.17$ & 25.75 & - & - \\
SUB3-3 & $12: 25: 05.471$ & $15: 16: 30.21$ & 26.13 & - & - \\
SUB3-4 & $12: 25: 05.812$ & $15: 16: 28.84$ & 26.25 & - & 28.10 \\
SUB3-5* & $12: 24: 26.934$ & $15: 15: 43.10$ & 26.31 & 25.46 & 27.30 \\
SUB3-6* & $12: 25: 05.369$ & $15: 23: 04.51$ & 26.46 & 25.46 & 27.66 \\
SUB3-7* & $12: 23: 32.742$ & $15: 16: 58.09$ & 26.49 & - & 27.06 \\
SUB3-8 & $12: 25: 15.651$ & $15: 27: 13.45$ & 26.57 & - & 26.86 \\
SUB3-9 & $12: 25: 00.771$ & $15: 15: 52.94$ & 26.99 & - & 26.72 \\
SUB3-10 & $12: 24: 35.389$ & $15: 08: 58.35$ & 27.52 & - & 28.72 \\
SUB3-11 & $12: 23: 40.561$ & $15: 09: 28.04$ & 27.53 & - & - \\
\hline
\end{tabular}

Objects with (*) are ICPNe.

\section{References}

Aguerri, J. A. L., Gerhard, O. E., Arnaboldi, M., et al. 2005, AJ, 129, 2585

Aguerri, J. A. L., Castro-Rodríguez, N., Napolitano, N., Arnaboldi, M., \& Gerhard, O. 2006, A\&A, 457, 771

Alcala, J. M., Radovich, M., Silvotti, R., et al. 2002, in SPIE Conf. Ser. 4836, ed. J. A. Tyson, \& S. Wolff, 406

Arnaboldi, M., Freeman, K. C., Mendez, R. H., et al. 1996, ApJ, 472, 145 Arnaboldi, M., Aguerri, J. A. L., Napolitano, N. R., et al. 2002, AJ, 123, 760 Arnaboldi, M., Freeman, K. C., Okamura, S., et al. 2003, AJ, 125, 514

Arnaboldi, M., Gerhard, O., Aguerri, J. A. L., et al. 2004, ApJ, 614, L33 Arnaboldi, M., Doherty, M., Gerhard, O., et al. 2008, ApJ, 674, L17

Bekki, K., Couch, W. J., \& Shioya, Y. 2002, ApJ, 577, 651

Bertin, E., \& Arnouts, S. 1996, A\&AS, 117, 393

Binggeli, B., Tammann, G. A., \& Sandage, A. 1987, AJ, 94, 251

Binggeli, B., Popescu, C. C., \& Tammann, G. A. 1993, A\&AS, 98, 275

Buzzoni, A., Arnaboldi, M., \& Corradi, R. L. M. 2006, MNRAS, 368, 877

Castro-Rodríguez, N., Aguerri, J. A. L., Arnaboldi, M., et al. 2003, A\&A, 405, 803

Ciardullo, R., Jacoby, G. H., Ford, H. C., \& Neill, J. D. 1989, ApJ, 339, 53

Ciardullo, R., Feldmeier, J. J., Jacoby, G. H., et al. 2002a, ApJ, 577, 31

Ciardullo, R., Feldmeier, J. J., Krelove, K., Jacoby, G. H., \& Gronwall, C. 2002b, ApJ, 566, 784

Ciardullo, R., Sigurdsson, S., Feldmeier, J. J., \& Jacoby, G. H. 2005, ApJ, 629, 499

Coccato, L., Gerhard, O., Arnaboldi, M., et al. 2009, MNRAS, 217

Colless, M., Ellis, R. S., Taylor, K., \& Hook, R. N. 1990, MNRAS, 244, 408

Doherty, M., Arnaboldi, M., Das, P., et al. 2009, A\&A, 502, 771

Durrell, P. R., Ciardullo, R., Feldmeier, J. J., Jacoby, G. H., \& Sigurdsson, S. 2002, ApJ, 570, 119

Feldmeier, J. J., Ciardullo, R., \& Jacoby, G. H. 1998, ApJ, 503, 109

Feldmeier, J. J., Mihos, J. C., Morrison, H. L., Rodney, S. A., \& Harding, P. 2002, ApJ, 575, 779

Feldmeier, J. J., Ciardullo, R., Jacoby, G. H., \& Durrell, P. R. 2003, ApJS, 145, 65

Feldmeier, J. J., Ciardullo, R., Jacoby, G. H., \& Durrell, P. R. 2004a, ApJ, 615, 196

Feldmeier, J. J., Mihos, J. C., Morrison, H. L., et al. 2004b, ApJ, 609, 617

Ferguson, H. C., Tanvir, N. R., \& von Hippel, T. 1998, Nature, 391, 461

Freeman, K. C., Arnaboldi, M., Capaccioli, M., et al. 2000, in Dynamics of Galaxies: from the Early Universe to the Present, ed. F. Combes, G. A. Mamon, \& V. Charmandaris, ASP Conf. Ser., 197, 389

Fukugita, M., Hogan, C. J., \& Peebles, P. J. E. 1998, ApJ, 503, 518

Gawiser, E., Francke, H., Lai, K., et al. 2007, ApJ, 671, 278

Gerhard, O., Arnaboldi, M., Freeman, K. C., \& Okamura, S. 2002, ApJ, 580, L121

Gerhard, O., Arnaboldi, M., Freeman, K. C., et al. 2005, ApJ, 621, L93

Gerhard, O., Arnaboldi, M., Freeman, K. C., et al. 2007, A\&A, 468, 815

Gronwall, C., Ciardullo, R., Hickey, T., et al. 2007, ApJ, 667, 79

Gunn, J. E., \& Gott, J. R. I. 1972, ApJ, 176, 1

Hammer, F., Flores, H., Lilly, S. J., et al. 1997, ApJ, 481, 49

Hogg, D. W., Cohen, J. G., Blandford, R., \& Pahre, M. A. 1998, ApJ, 504, 622

Jacoby, G. H. 1989, ApJ, 339, 39

Kormendy, J., Fisher, D. B., Cornell, M. E., \& Bender, R. 2009, ApJS, 182, 216

Krick, J. E. \& Bernstein, R. A. 2007, AJ, 134, 466

Kudritzki, R.-P., Méndez, R. H., Feldmeier, J. J., et al. 2000, ApJ, 536, 19 
Larson, R. B., Tinsley, B. M. \& Caldwell, C. N. 1980, ApJ, 237, 692

Merritt, D. 1984, ApJ, 276, 26

Mihos, C. J., Janowiecki, S., Feldmeier, J. J., Harding, P., \& Morrison, H. 2009, ApJ, 698, 1879

Mihos, J. C. 2004, in Clusters of Galaxies: Probes of Cosmological Structure and Galaxy Evolution, ed. J. S. Mulchaey, A. Dressler, \& A. Oemler, 277

Mihos, J. C., Harding, P., Feldmeier, J., \& Morrison, H. 2005, ApJ, 631, L41

Miyazaki, S., Komiyama, Y., Sekiguchi, M., et al. 2002, PASJ, 54, 833

Moore, B., Katz, N., Lake, G., Dressler, A., \& Oemler, A. 1996, Nature, 379, 613

Murante, G., Arnaboldi, M., Gerhard, O., et al. 2004, ApJ, 607, L83

Murante, G., Giovalli, M., Gerhard, O., et al. 2007, MNRAS, 377, 2

Napolitano, N. R., Pannella, M., Arnaboldi, M., et al. 2003, ApJ, 594, 172

Okamura, S., Yasuda, N., Arnaboldi, M., et al. 2002, PASJ, 54, 883

Ouchi, M., Shimasaku, K., Furusawa, H., et al. 2003, ApJ, 582, 60

Quilis, V., Moore, B., \& Bower, R. 2000, Science, 288, 1617

Ramella, M., Biviano, A., Pisani, A., et al. 2007, A\&A, 470, 39
Rauch, M., Haehnelt, M., Bunker, A., et al. 2008, ApJ, 681, 856 Richstone, D. O., \& Malumuth, E. M. 1983, ApJ, 268, 30

Rudick, C. S., Mihos, J. C., \& McBride, C. 2006, ApJ, 648, 936

Rudick, C. S., Christopher Mihos, J., Frey, L. H., \& McBride, C. K. 2009, ApJ, 699, 1518

Solanes, J. M., Sanchis, T., Salvador-Solé, E., Giovanelli, R., \& Haynes, M. P. 2002, AJ, 124, 2440

Sommer-Larsen, J., Romeo, A. D., \& Portinari, L. 2005, MNRAS, 357, 478

Theuns, T., \& Warren, S. J. 1997, MNRAS, 284, L11

West, M. J., Jones, C., \& Forman, W. 1995, ApJ, 451, L5+

White, S. D. M., \& Rees, M. J. 1978, MNRAS, 183, 341

Willman, B., Governato, F., Wadsley, J., \& Quinn, T. 2004, MNRAS, 355, 159

Williams, B. F., Ciardullo, R., Durrell, P. R., et al. 2007, ApJ, 656, 756

Yan, H., Hathi, N. P., \& Windhorst, R. A. 2008, ApJ, 675, 136

Yasuda, N., Fukugita, M., \& Okamura, S. 1997, ApJS, 108, 417

Zibetti, S., White, S. D. M., Schneider, D. P., \& Brinkmann, J. 2005, MNRAS, 358,949 